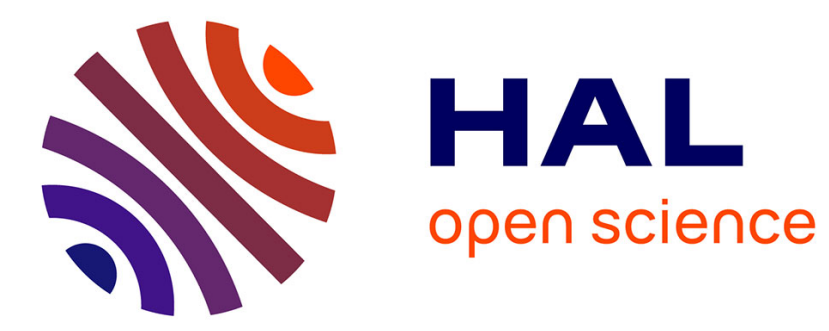

\title{
Multi-leg integrable ladder models
}

Daniel Arnaudon, Ara Sedrakyan, Tigran Sedrakyan

\section{To cite this version:}

Daniel Arnaudon, Ara Sedrakyan, Tigran Sedrakyan. Multi-leg integrable ladder models. Nuclear Physics B, 2004, B676, pp.615. hal-00003183

\section{HAL Id: hal-00003183 \\ https://hal.science/hal-00003183}

Submitted on 27 Oct 2004

HAL is a multi-disciplinary open access archive for the deposit and dissemination of scientific research documents, whether they are published or not. The documents may come from teaching and research institutions in France or abroad, or from public or private research centers.
L'archive ouverte pluridisciplinaire HAL, est destinée au dépôt et à la diffusion de documents scientifiques de niveau recherche, publiés ou non, émanant des établissements d'enseignement et de recherche français ou étrangers, des laboratoires publics ou privés. 


\title{
Multi-leg integrable ladder models
}

\author{
D. Arnaudon ${ }^{1}$, A. Sedrakyan ${ }^{2}$, T. Sedrakyan ${ }^{3}$, \\ Laboratoire d'Annecy-le-Vieux de Physique Théorique LAPTH \\ CNRS, UMR 5108, associée à l'Université de Savoie \\ BP 110, F-74941 Annecy-le-Vieux Cedex, France
}

\begin{abstract}
We construct integrable spin chains with inhomogeneous periodic disposition of the anisotropy parameter. The periodicity holds for both auxiliary (space) and quantum (time) directions. The integrability of the model is based on a set of coupled YangBaxter equations. This construction yields $P$-leg integrable ladder Hamiltonians. We analyse the corresponding quantum group symmetry and present Algebraic Bethe Ansatz $(A B A)$ solution.
\end{abstract}

LAPTH-944/02

hep-th/0210087

\footnotetext{
1e-mail:arnaudon@lapp.in2p3.fr

2e-mail:sedrak@lx2.yerphi.am, Permanent address: Yerevan Physics Institute, Armenia

${ }^{3}$ e-mail:tigrans@moon.yerphi.am, Permanent address: Yerevan Physics Institute, Armenia
} 


\section{Introduction}

In an attempt to formulate the Chalker-Coddington model in the action formalism with coherent states [1], one is led to the use of Manhattan Lattice $M L$ [2, 3]. One has also to consider the $R$-matrices of the $X X$ model with alternating shifts of the spectral parameter. Therefore the idea of a $\mathbb{Z}_{2}$ staggering of the spaces along the chain and time directions naturally rises up.

Initiating this fact in a series of articles [4, 5, 6, 7] the authors have proposed a technique for constructing integrable models where the model parameter $\Delta$ (the anisotropy parameter of the $X X Z$ and anisotropic $t-J$ models) have a staggered disposition of the sign along both chain and time directions. Due to the staggered shift of the spectral parameter also considered there, these models have led to Hamiltonians formulated on two leg zig-zag quasi-one dimensional chains.

The technique is based on an appropriate modification of the Yang-Baxter equations $(Y B E)$ ([8, 9]) for the $R$-matrices which are the conditions for commutativity of the transfer matrices at different values of the spectral parameter. The transfer matrix is defined as the staggered product of $R$-matrices, which have staggered sign of the anisotropy parameter $\Delta$ and staggered shift of the spectral parameter of the model along the chain. The shift of spectral parameter in a product of $R$-matrices was considered in earlier works of several authors [10, 11], by use of which they have analysed the ordinary $X X Z$ model in the infinite limit of the spectral parameter. Later, in the article [12], this modified transfer matrices has been used in order to construct integrable models on the ladder.

Integrable models on ladders were also constructed in various articles [13, 14, 15, 16, 17] but our construction essentially differs from those because it contains an inhomogeneity of the anisotropy parameter along the chain.

In the article [7] we have analysed the quantum group structure behind of our construction and have shown that it is a tensor product $\mathcal{W} \otimes \mathcal{U}_{q, i}(g l(2))$ of a Weyl algebra and a two parameter $(q$ and $i)$ deformation of $\mathcal{U}(g l(2))$.

In the present article we go further in this direction by presenting the corresponding modification of our technique to consider and construct $P$-leg integrable models, based on the $S L(2)$ group (the generalisation of the 2-leg $X X Z$ case).

In the Section 2 we present the basic definitions of our construction, namely the $R$ matrices, the shifts of the spectral parameter, the transfer matrix and the corresponding modified $Y B E$ as the conditions of commutativity of the transfer matrix for different values of the spectral parameters. We also present the solution of the coupled $Y B E$ for the $X X Z$ case.

In the Section 3 we calculate the Hamiltonian of our model, showing that it can be considered on the $P$-leg ladder. We first prove that our transfer matrix at zero value of the spectral parameter is proportional to the identity operator (in the braid formalism). The Hamiltonian, as usual, is defined as the linear term in the spectral parameter expansion of the transfer matrix. 
In Section 4 we analyse the quantum group structure behind of our Yang-Baxter equations. We show that the algebra defined by the RLL relations [18] derived from the set of coupled Yang-Baxter equations can be regarded as direct product of a Weyl algebra and the $q$-deformed $\mathcal{U}_{q}(\operatorname{sl}(2))$.

In Section 5 we present Algebraic Bethe Ansatz solution of our model.

\section{Basic Definitions and the Yang-Baxter equations}

Let us now consider $\mathbb{Z}_{P}$ graded quantum $V_{j, \rho}(v)$ (with $j=1, \ldots, L$ as a chain index) and auxiliary $V_{a, \sigma}(u)$ spaces, where $\rho, \sigma=0,1, \ldots, P-1$ are the grading indices. We consider $R$-matrices which act on the direct product of spaces $V_{a, \sigma}(u)$ and $V_{j, \rho}(v),(\sigma, \rho=$ $0,1, \ldots, P-1)$, mapping them on the intertwined direct product of $V_{a, \sigma+1}(u)$ and $V_{j, \rho+1}(v)$ spaces (we impose periodic boundary conditions $P \equiv 0$ )

$$
R_{a j, \sigma \rho}(u, v): \quad V_{a, \sigma}(u) \otimes V_{j, \rho}(v) \rightarrow V_{j, \rho+1}(v) \otimes V_{a, \sigma+1}(u) .
$$

Definition 2.1 We introduce two transmutation operations $\iota_{1}$ and $\iota_{2}$ with the property $\iota_{1}^{P}=\iota_{2}^{P}=i d$ for the quantum and auxiliary spaces correspondingly, and mark the operators $R_{a j, \sigma \rho}$ as follows

$$
\begin{array}{rllll}
R_{a j, 00} & \equiv R_{a j}, & R_{a j, 01} \equiv R_{a j}^{\iota_{1}} & \ldots & R_{a j, 0(P-1)} \equiv R_{a j}^{\iota_{1}^{P-1}}, \\
R_{a j, 10} & \equiv R_{a j}^{\iota_{2}}, & R_{a j, 11} \equiv R_{a j}^{\iota_{1} \iota_{2}} & \ldots & R_{a j, 1(P-1)} \equiv R_{a j}^{\iota_{2} \iota_{1}^{P-1}}, \\
\vdots & & & & \\
R_{a j,(P-1) 0} & \equiv R_{a j}^{l_{2}^{P-1}}, & R_{a j,(P-1) 1} \equiv R_{a j}^{\iota_{1} \iota_{2}^{P-1}} \ldots & R_{a j,(P-1)(P-1)} \equiv R_{a j}^{\ell_{1}^{P-1} \iota_{2}^{P-1}} .
\end{array}
$$

The introduction of the $\mathbb{Z}_{P}$ grading of quantum spaces in time direction means, that we have now $P$ monodromy operators $T_{\rho}, \rho=0,1, \ldots, P-1$, which act on the space

$$
V_{\rho}(u)=\prod_{j=1}^{L} V_{j, \rho}(u)
$$

by mapping it on $V_{\rho+1}(u)=\prod_{j=1}^{P} V_{j, \rho+1}(u)$

$$
T_{\rho}(v-u) \quad: V_{\rho}(u) \rightarrow V_{\rho+1}(u), \quad \rho=0,1, . . P-1 .
$$

It is clear now, that the monodromy operator of the model, which is defined by translational invariance in $P$-steps in the time direction and determines the partition function, is the product of $P$ monodromy operators

$$
T(u)=\prod_{\sigma=0}^{P-1} T_{\sigma}(u) .
$$


The $\mathbb{Z}_{P}$ grading of the auxiliary spaces along the chain direction besides the $\iota$ operations defined upper contained also a shift of the spectral parameter. Let us define the following shift operations on the spectral parameter $u$

$$
\bar{u}=\bar{u}^{(1)}=\theta+\epsilon u, \quad \cdots, \quad \bar{u}^{(k)}=\theta+\epsilon \bar{u}^{(k-1)} \quad k=0,1,2, \cdots, P,
$$

where $\epsilon=e^{2 \pi i / P}$ is the $P^{t h}$ root of unity and $\theta$ is an additional model parameter. It is easy to prove that $\bar{u}^{(P)}=\bar{u}^{(0)}=u$.

By use of this shift operations we define the $T_{\sigma}(u)$ monodromy matrices according to the following

Definition 2.2 We define the monodromy operators $T_{\sigma}(u), \sigma=0,1,2 \ldots, P-1$ as a product of the $R_{a j, \sigma \rho}(u)$ matrices:

$$
\begin{array}{r}
T_{0}(u)=\prod_{j=0}^{L / P}\left(R_{a, P j}(u) R_{a, P j+1}^{\iota_{2}}(\bar{u}) \cdots R_{a, P j+P-1}^{\iota_{2}^{P-1}}\left(\bar{u}^{(P-1)}\right)\right), \\
T_{1}(u)=\prod_{j=0}^{L / P}\left(R_{a, P j}^{\iota_{1}}(\bar{u}) R_{a, P j+1}^{\iota_{1} \iota_{2}}\left(\bar{u}^{(2)}\right) \cdots R_{a, P j+P-1}^{\iota_{1} 1_{2}^{P-1}}\left(\bar{u}^{(P)}\right)\right), \\
T_{p}(u)=\prod_{j=0}^{L / P}\left(R_{a, P j}^{\iota_{1}^{p}}\left(\bar{u}^{(p)}\right) R_{a, P j+1}^{\iota_{1}^{p} \iota_{2}}\left(\bar{u}^{(p+1)}\right) \cdots R_{a, P j+P-1}^{\iota_{1}^{p} l_{2}^{P-1}}\left(\bar{u}^{(p+P-1)}\right)\right), \\
p=0,1 \ldots, P-1 .
\end{array}
$$

We will consider this monodromy operators in this paper and analyse their commutativity next.

As it is well known in Bethe Ansatz Technique [8, 9], a sufficient condition for the commutativity of transfer matrices $\tau_{p}(u)=\operatorname{Tr} T_{p}(u)$ with different spectral parameters is the Yang-Baxter equation $(Y B E)$. In order to have a commutativity of transfer matrices (2.5) for different values of the spectral parameter we demand the commutativity of transfer matrices (2.7). Then the standard so called railway arguments yields the following set of $P$ equations

$$
\begin{aligned}
R_{12}(\bar{u}-v) R_{13}^{\iota_{1}}(\bar{u}) R_{23}(v) & =R_{23}^{\iota_{1}}(v) R_{13}(\bar{u}) \tilde{R}_{12}^{(1)}(\bar{u}-v) \\
\tilde{R}_{12}^{(1)}(\bar{u}-v) R_{13}^{\iota_{1} \iota_{2}}\left(\bar{u}^{(2)}\right) R_{23}^{\iota_{2}}(\bar{v}) & =R_{23}^{\iota_{1} \iota_{2}}(\bar{v}) R_{13}^{\iota_{2}}\left(\bar{u}^{(2)}\right) \tilde{R}_{12}^{(2)}\left(\bar{u}^{(2)}-\bar{v}\right), \\
& \vdots \\
\tilde{R}_{12}^{(P-1)}\left(\bar{u}^{(P)}-\bar{v}^{(P-1)}\right) R_{13}^{\iota_{1} \iota_{2}^{P-1}}\left(\bar{u}^{(P)}\right) R_{23}^{\iota^{2}-1}\left(\bar{v}^{(P-1)}\right) & =R_{23}^{\iota_{1} \iota_{2}^{P-1}}\left(\bar{v}^{(P-1)}\right) R_{13}^{\iota_{2} P-1}\left(\bar{u}^{(P)}\right) R_{12}(\bar{u}-v) .
\end{aligned}
$$


It can be seen easily that, defining ${ }^{\iota_{2}}$ and $\tilde{R}^{(p)}$ as

$$
\begin{aligned}
R^{\iota_{2}}(u) & =R^{\iota_{1}}\left(\epsilon^{-1} u\right), \\
\tilde{R}^{(p)}(u-v) & =R^{\iota_{1}^{p}}(u-v), \quad p=1, \ldots, P-1,
\end{aligned}
$$

all the equations (2.8) are compatible and reducing to the first one.

Now, in order to solve this equation for the $R(u)$ above, we follow a procedure which is the inverse of the Baxterisation (debaxterisation) [19]. $\mathcal{R}(u)$ can indeed be written

$$
R_{12}(u)=\frac{1}{2 i}\left(z R_{12}-z^{-1} R_{21}^{-1}\right)
$$

with $z=e^{i u}$ and the constant $R_{12}$ and $R_{21}^{-1}$ matrices are spectral parameter independent. Then the Yang-Baxter equations (2.8) for the spectral parameter dependent $R$-matrix $R(u)$ and $R^{\iota_{1}}(u)$ are equivalent to the following equations for the constant $R$-matrices

$$
\begin{aligned}
R_{12} R_{13}^{\iota_{1}} R_{23} & =R_{23}^{\iota_{1}} R_{13} R_{12}^{\iota_{1}} \\
R_{12}^{\iota_{1}} R_{13} R_{23}^{\iota_{1}} & =R_{23} R_{13}^{\iota_{1}} R_{12} \\
R_{12}\left(R_{31}^{\iota_{1}}\right)^{-1} R_{23}-\left(R_{21}\right)^{-1} R_{13}^{\iota_{1}}\left(R_{32}\right)^{-1} & =R_{23}^{\iota_{1}}\left(R_{31}\right)^{-1} R_{12}^{\iota_{1}}-\left(R_{32}^{\iota_{1}}\right)^{-1} R_{13}\left(R_{21}^{\iota_{1}}\right)^{-1} \\
R_{12}^{\iota_{1}}\left(R_{31}\right)^{-1} R_{23}^{\iota_{1}}-\left(R_{21}^{\iota_{1}}\right)^{-1} R_{13}\left(R_{32}^{\iota_{1}}\right)^{-1} & =R_{23}\left(R_{31}^{\iota_{1}}\right)^{-1} R_{12}-\left(R_{32}\right)^{-1} R_{13}^{\iota_{1}}\left(R_{21}\right)^{-1} .
\end{aligned}
$$

If this modified YBE's have a solution, then one can formulate new integrable models on the basis of existing ones. We will hereafter give solutions of these YBE's based on $\mathcal{U}_{q}(\operatorname{sl}(2)) R$-matrices.

A solution of (2.11)-(2.14) is then given by

$$
\begin{aligned}
R & =\left(\begin{array}{llll}
q & 0 & 0 & 0 \\
0 & 1 & 0 & 0 \\
0 & q-q^{-1} & 1 & 0 \\
0 & 0 & 0 & q
\end{array}\right), \\
R^{\iota_{1}^{p}} & =\left(\begin{array}{llll}
q & 0 & 0 & 0 \\
0 & \epsilon^{p} & 0 & 0 \\
0 & q-q^{-1} & \epsilon^{-p} & 0 \\
0 & 0 & 0 & q
\end{array}\right),
\end{aligned}
$$

where (2.15) is the usual $R$-matrix of $\mathcal{U}_{q}(g l(2))$.

The solution obtained in [4] in connection with the staggered XXZ model for $P=2$, can then be generalised to a solution of (2.8) given by

$$
R(u)=\left(\begin{array}{llll}
\sin (\lambda+u) & 0 & 0 & 0 \\
0 & \sin (u) & e^{-i u} \sin (\lambda) & 0 \\
0 & e^{i u} \sin (\lambda) & \sin (u) & 0 \\
0 & 0 & 0 & \sin (\lambda+u)
\end{array}\right)
$$




$$
R^{\iota_{1}^{p}}(u)=\left(\begin{array}{llll}
\sin (\lambda+u) & 0 & 0 & 0 \\
0 & \epsilon^{p} \cdot \sin (u) & e^{-i u} \sin (\lambda) & 0 \\
0 & e^{i u} \sin (\lambda) & \epsilon^{-p} \cdot \sin (u) & 0 \\
0 & 0 & 0 & \sin (\lambda+u)
\end{array}\right)
$$

(Notice that we introduced here the off-diagonal factors $e^{i u}$ and $e^{-i u}$ not present in [4 to allow the decomposition (2.10). They are nothing more than a rescaling of the states or a simple gauge transformation.)

Following the technique developed in the article [20] one can fermionise the $R$-matrix of the $X X Z$ model by using for the $V_{a, \sigma}$ and $V_{j, \rho}$ spaces the Fock space of the Fermi fields $c_{i}, c_{i}^{+}$with basis vectors $|0\rangle_{i}$ and $|1\rangle_{i}$, for which

$$
\left(X_{i}\right)_{a}^{a^{\prime}}=\left(\begin{array}{ll}
1-n_{i} & c_{i}^{+} \\
c_{i} & n_{i}
\end{array}\right)
$$

is the Hubbard operator. Then the fermionic $R$-operator is defined by use of (2.17)

$$
\begin{aligned}
R_{a j} & =(-1)^{p\left(a_{1}\right) p\left(j_{2}\right)}\left(R_{a j}\right)_{a_{1} j_{1}}^{a_{2} j_{2}} X_{a_{2}}^{a_{1}} X_{j_{2}}^{j_{1}} \\
& =a(u)\left[-n_{j} n_{k}+\left(1-n_{j}\right)\left(1-n_{k}\right)\right]+b(u)\left[n_{j}\left(1-n_{k}\right)+\left(1-n_{j}\right) n_{k}\right] \\
& +c(u)\left[c_{j}^{+} c_{k}+c_{k}^{+} c_{j}\right]
\end{aligned}
$$

where $a(u)=\sin (\lambda+u), \quad b(u)=\sin (u), \quad c(u)=\sin (\lambda)$.

The corresponding expressions for the $R_{1}^{\iota_{1}^{k}}(k=1, \cdots, P-1)$ operators are

$$
\begin{aligned}
R_{a j}^{\iota_{1}^{k}} & =a(u)\left[-n_{j} n_{k}+\left(1-n_{j}\right)\left(1-n_{k}\right)\right]+b(u)\left[\epsilon^{-k} n_{j}\left(1-n_{k}\right)+\epsilon^{k}\left(1-n_{j}\right) n_{k}\right] \\
& +c(u)\left[c_{j}^{+} c_{k}+c_{k}^{+} c_{j}\right] .
\end{aligned}
$$

\section{Hamiltonian for the $P$-leg model}

Usually in order to calculate the Hamiltonian in an homogeneous chain we should expand the transfer matrix around the point where it becomes identity operator (in the braid formalism). But since we have different shifts of the spectral parameter in our model it is impossible to find a value of the spectral parameter such that all the $R$ matrices become identity. Therefore we choose $u=0$ as an expansion point, where only some of the $R$-matrices become identity, while the others contain scatterings. This is the origin of the appearance of the next to nearest neighbour interaction terms $(N N N)$ in the Hamiltonian and of the formulation of the model on the P-leg quasi-one dimensional chain.

We should calculate the transfer matrix at the point $u=0$ and the linear term of its expansion over $u$. 
Proposition 3.1 The transfer matrix $\check{T}(0)$ in the braid formalism is proportional to the identity operator

$$
\check{T}(0) \simeq \mathbb{I}
$$

Proof: The proof is based on the use of YBE at the point $u=0$ and graphically represented in Figure 1 in the $P=3$ case.

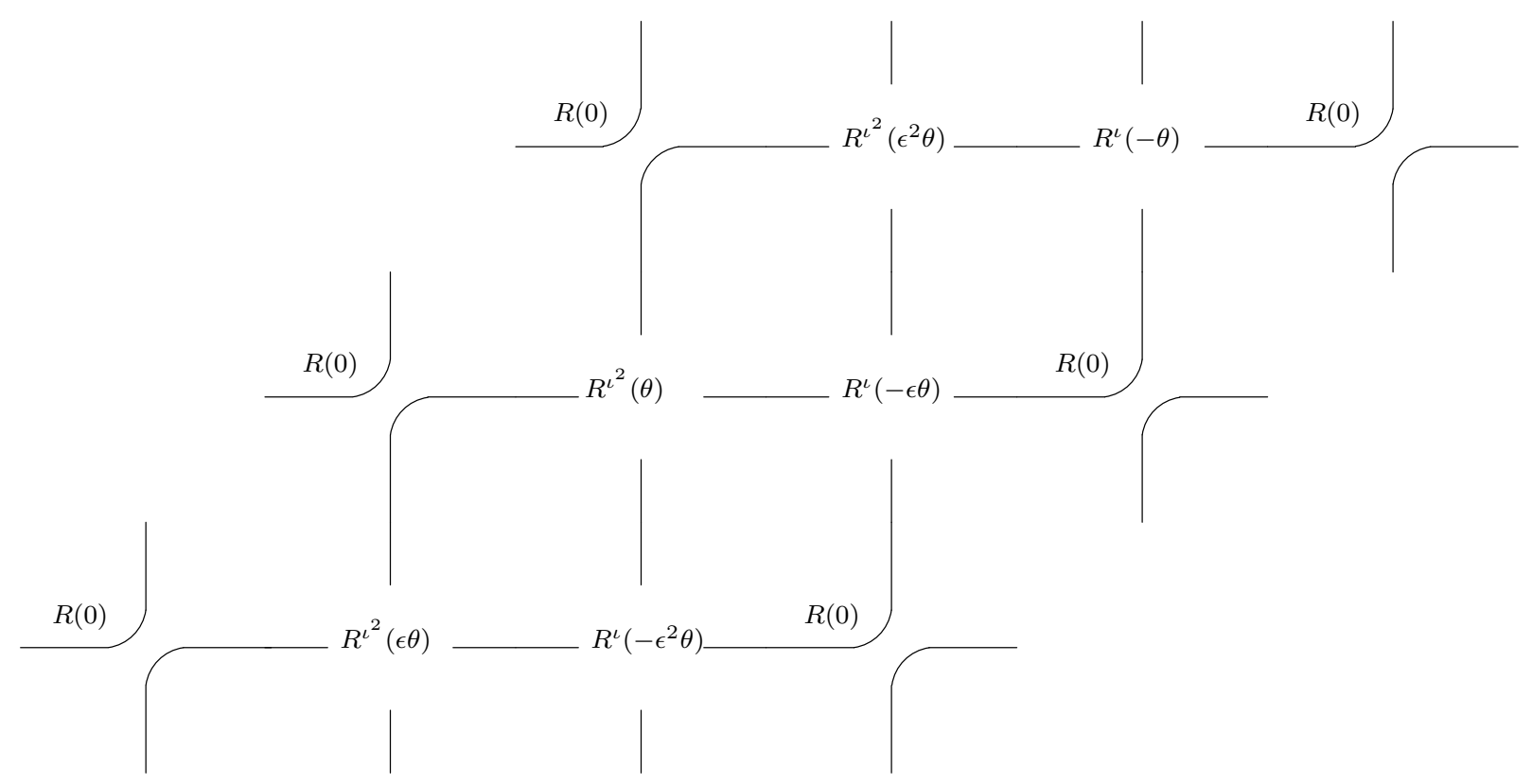

Figure 1: Transfer matrix at $u=0$ for $P=3$

$T(u)$ is a product of $R$-matrices that can be written in several ways, simply using the trivial commutations of matrices that have no indices in common. On way to write $T(0)$ is

$$
T(0)=\prod_{j=2}^{P} \prod_{i=1}^{j-1} R_{i j}^{\iota^{P-j+i}}\left(\left(\epsilon^{-j}-\epsilon^{-i}\right) \theta^{\prime}\right) \prod_{j=1}^{P-1} \prod_{i=j+1}^{P} R_{i j}^{L^{P-j+i}}\left(\left(\epsilon^{-j}-\epsilon^{-i}\right) \theta^{\prime}\right)
$$

where $\theta^{\prime}=\theta /(1-\epsilon)$ and $\iota \equiv \iota_{1}$. The first terms of this product are $R_{12} R_{13} R_{23}$ (with convenient arguments and powers of $\iota$ ), for which we use the Yang-Baxter equation. $R_{12}$ meets then $R_{14} R_{34}$ and $R_{13}$ meets $R_{14} R_{34}$. And so on, until all the $R_{1 j}$ matrices are in the middle (between the double products), so that

$$
T(0)=\prod_{j=3}^{P} \prod_{i=2}^{j-1} R_{i j}^{\iota^{P-j+i-1}}\left(\left(\epsilon^{-j}-\epsilon^{-i}\right) \theta^{\prime}\right) \overleftarrow{\prod_{j=2, . ., k}} R_{1 j}^{\iota^{P+j-1}} \prod_{j=1}^{P-1} \prod_{i=j+1}^{P} R_{i j}^{\iota^{P-j+i}}\left(\left(\epsilon^{-j}-\epsilon^{-i}\right) \theta^{\prime}\right)
$$


The $R_{i 1}$ terms present in the last double product precisely cancels the product $\prod_{j=2, . ., k} R_{1 j}^{e^{P+j-1}}$ by unitarity property, so that

$$
T(0)=\prod_{j=3}^{P} \prod_{i=2}^{j-1} R_{i j}^{\iota^{P-j+i-1}}\left(\left(\epsilon^{-j}-\epsilon^{-i}\right) \theta^{\prime}\right) \prod_{j=2}^{P-1} \prod_{i=j+1}^{P} R_{i j}^{\iota^{P-j+i}}\left(\left(\epsilon^{-j}-\epsilon^{-i}\right) \theta^{\prime}\right)
$$

The powers of $\iota$ have changed in the remaining part. Following a straightforward recursion, we get

$$
T(0)=\prod_{j=l+1}^{P} \prod_{i=l}^{j-1} R_{i j}^{\iota^{P-j+i-l+1}}\left(\left(\epsilon^{-j}-\epsilon^{-i}\right) \theta^{\prime}\right) \prod_{j=l}^{P-1} \prod_{i=j+1}^{P} R_{i j}^{\ell^{P-j+i}}\left(\left(\epsilon^{-j}-\epsilon^{-i}\right) \theta^{\prime}\right)
$$

and finally $T(0)=1$ (up to some translations).

\section{Hamiltonian for the 3-leg model}

We will restricts ourselves to $P=3$ in the parts of this article concerned with the calculation of the Hamiltonian (the section concerned by the underlying algebra deals with general $P$ ).

The transfer matrix $T(u)=T_{0}(u) T_{1}(u) T_{2}(u)$ for $P=3$ is defined by formulae (2.7) containing only three lines

$$
\begin{aligned}
& T_{0}(u)=\prod_{j=0}^{L / 3}\left(R_{a, 3 j}(u) R_{a, 3 j+1}^{\iota_{2}}(\bar{u}) R_{a, 3 j+2}^{\iota_{2}^{2}}\left(\bar{u}^{(2)}\right)\right), \\
& T_{1}(u)=\prod_{j=0}^{L / 3}\left(R_{a, 3 j}^{\iota_{1}}(\bar{u}) R_{a, 3 j+1}^{\iota_{1} \iota_{2}}\left(\bar{u}^{(2)}\right) R_{a, 3 j+2}^{\iota_{1} \iota_{2}^{2}}(u)\right), \\
& T_{2}(u)=\prod_{j=0}^{L / 3}\left(R_{a, 3 j}^{\iota_{1}^{2}}\left(\bar{u}^{(2)}\right) R_{a, 3 j+1}^{\iota_{1}^{2} \iota_{2}}(u) R_{a, 3 j+2}^{\iota_{1}^{2} \iota_{2}^{2}}(\bar{u})\right) .
\end{aligned}
$$

For convenience let us use the graphical representations of the $R$-matrices as it is defined in the article [4] and represented in Figure 2.

Later we will pass to the so called coherent state basis for the $R$-matrices represented via Grassmann variables $\psi$, by which the corners of the square in the picture are labelled. The transfer matrix $T(u)=T_{0}(u) T_{1}(u) T_{2}(u)$ defined by formulae (2.7) contains only three lines and can be drawn as in Figure 3 .

In order to calculate the Hamiltonian one should take the expansion over the spectral parameter $u$ of the $R$-matrices in the expression (3.6) of the transfer matrix, and extract 


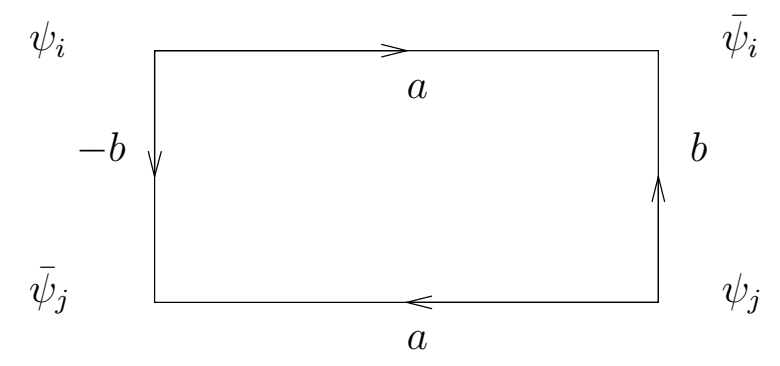

Figure 2: $R_{i j}$

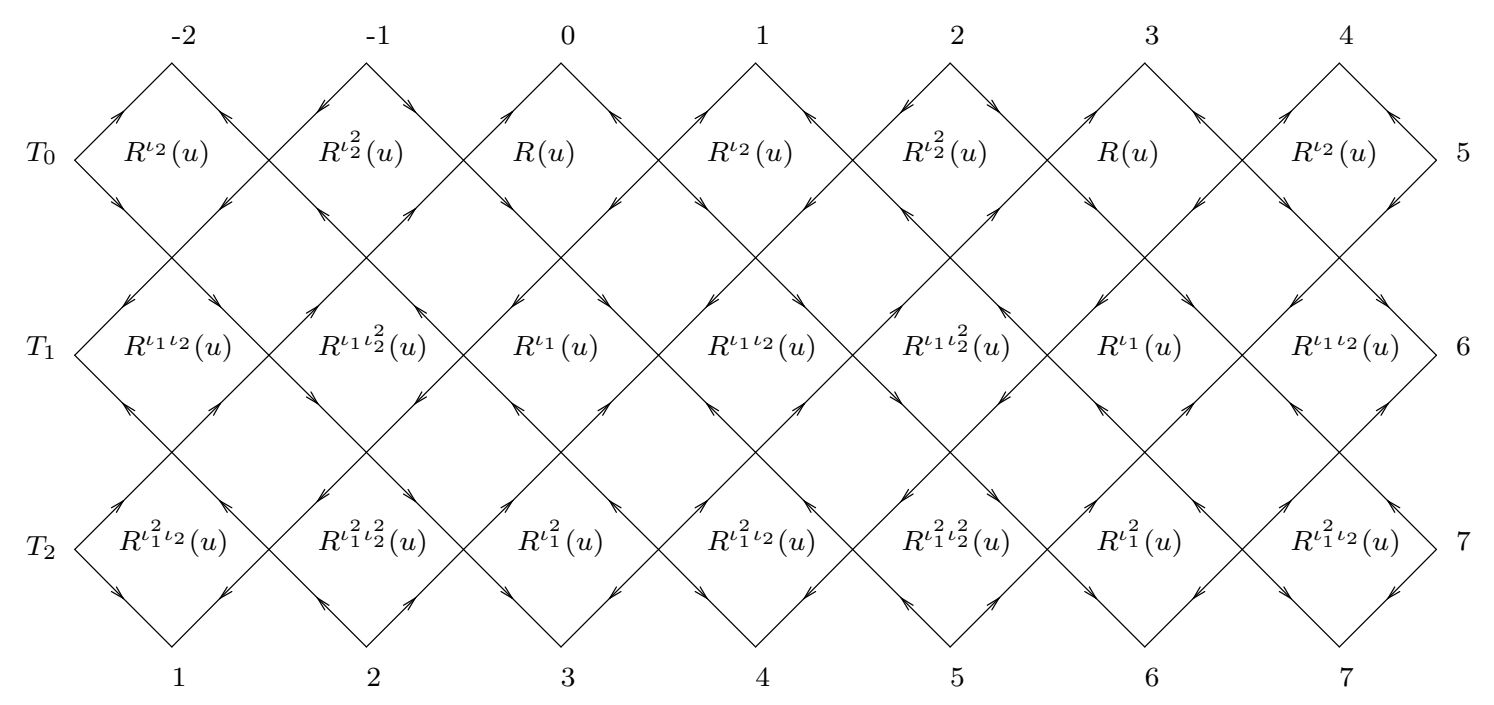

Figure 3: Triple row monodromy matrix 
the linear term. As one can see from Figure 3, we have nine $R$-matrices forming a block, by translation of which the whole transfer matrix can be reproduced. By use of $T(0) \sim \mathbb{I}$ proved before one can obtain the following expression for the contribution of one block to the Hamiltonian

$$
\begin{aligned}
& T(u)=a_{1} a_{2} \cdots a_{9}\left(\mathbb{I}+u \mathcal{H}_{01234}\right), \\
& \mathcal{H}_{01234}=\mathcal{H}_{01234}^{(1)}+\mathcal{H}_{01234}^{(2)}, \\
& \mathcal{H}_{01234}^{(1)}=H_{12}^{\iota_{1}^{2} \iota_{2}^{2}}\left(\check{R}_{12}^{\iota_{1}^{2} \iota_{2}^{2}}\right)^{-1}\left(\overline{0}^{(1)}\right)+\left(\check{R}_{23}^{\iota_{2}^{2}}\right)^{-1}\left(\overline{0}^{(2)}\right) H_{23}^{\iota_{2}^{2}} \\
& +\check{R}_{12}^{\iota_{1}^{2} \iota_{2}^{2}}\left(\overline{0}^{(1)}\right) \check{R}_{23}^{\iota_{1}^{2}}\left(\overline{0}^{(2)}\right) H_{12}^{\iota_{1}}\left(\check{R}_{12}^{\iota_{1}}\right)^{-1}\left(\overline{0}^{(1)}\right)\left(\check{R}_{23}^{\iota_{2}^{2}}\right)^{-1}\left(\overline{0}^{(2)}\right)\left(\check{R}_{12}^{\iota_{1}^{2} \iota_{2}^{2}}\right)^{-1}\left(\overline{0}^{(1)}\right) \\
& +\left(\check{R}_{23}^{\iota_{2}^{2}}\right)^{-1}\left(\overline{0}^{(2)}\right)\left(\check{R}_{12}^{\iota_{2}}\right)^{-1}\left(\overline{0}^{(1)}\right) H_{12}^{\iota_{2}} \check{R}_{23}^{\iota_{2}^{2}}\left(\overline{0}^{(2)}\right) \\
& +\check{R}_{12}^{l_{1}^{2} \iota_{2}^{2}}\left(\overline{0}^{(1)}\right) H_{23}^{\iota_{1}^{2}}\left(\check{R}_{23}^{l_{1}^{2}}\right)^{-1}\left(\overline{0}^{(2)}\right)\left(\check{R}_{12}^{\iota_{1}^{2} L_{2}^{2}}\right)^{-1}\left(\overline{0}^{(1)}\right) \\
& +\left(\check{R}_{23}^{\iota_{2}^{2}}\right)^{-1}\left(\overline{0}^{(2)}\right)\left(\check{R}_{12}^{\iota_{2}}\right)^{-1}\left(\overline{0}^{(1)}\right)\left(\check{R}_{23}^{\iota_{1} \iota_{2}}\right)^{-1}\left(\overline{0}^{(2)}\right) H_{23}^{\iota_{1} \iota_{2}} \check{R}_{12}^{\iota_{2}}\left(\overline{0}^{(1)}\right) \check{R}_{23}^{\iota_{2}^{2}}\left(\overline{0}^{(2)}\right) \text {, } \\
& \mathcal{H}_{01234}^{(2)}=\check{R}_{12}^{\iota_{1}^{2} \iota_{2}^{2}}\left(\overline{0}^{(1)}\right) \check{R}_{23}^{\iota_{1}^{2}}\left(\overline{0}^{(2)}\right) H_{34}^{\iota_{1}^{2} \iota_{2}}\left(\check{R}_{34}^{\iota_{1}^{2} \iota_{2}}\right)^{-1}(0)\left(\check{R}_{23}^{\iota_{1}^{2}}\right)^{-1}\left(\overline{0}^{(2)}\right)\left(\check{R}_{12}^{\iota_{1}^{2} \iota_{2}^{2}}\right)^{-1}\left(\overline{0}^{(1)}\right) \\
& +\left(\check{R}_{23}^{\iota_{2}^{2}}\right)^{-1}\left(\overline{0}^{(2)}\right)\left(\check{R}_{34}^{\iota_{1} \iota_{2}^{2}}\right)^{-1}(0) H_{34}^{\iota_{1} \iota_{2}^{2}} \check{R}_{23}^{\iota_{2}^{2}}\left(\overline{0}^{(2)}\right) \\
& +\quad\left(\check{R}_{12}^{\iota_{1}^{2} \iota_{2}^{2}}\right)\left(\overline{0}^{(1)}\right) H_{01}^{\iota_{1} \iota_{2}^{2}}\left(\check{R}_{01}^{\iota_{1} \iota_{2}^{2}}\right)^{-1}(0)\left(\check{R}_{12}^{\iota_{1}^{2} \iota_{2}^{2}}\right)^{-1}\left(\overline{0}^{(1)}\right) \\
& \left.+\left(\check{R}_{23}^{\iota_{2}^{2}}\right)^{-1}\left(\overline{0}^{(2)}\right)\left(\check{R}_{12}^{\iota_{2}}\right)^{-1}(\overline{0}) H_{34} \check{R}_{12}^{\iota_{2}}\right)\left(\overline{0} \check{R}_{23}^{\iota_{2}^{2}}\left(\overline{0}^{(2)}\right)\right.
\end{aligned}
$$

where the notation $\overline{0}^{(k)}, k=1,2$ means $\bar{u}^{(k)}$ at $u=0$ defined by the formula (2.6). In this expression $H_{i j}^{\iota_{1}^{p} \iota_{2}^{q}}$ are the linear terms of the expansions of the corresponding $R$-matrices and we will write down their explicit expressions later.

The formulae (3.7) and (3.8) show that we need the expressions of nine $R$-matrices, as well as their derivatives, at $u=0$. Instead of using in the future the long upper scripts $\iota_{1}^{k} \iota_{2}^{p}$ it looks convenient to introduce the following numeration for those nine $R$-matrices (see Figure 3)

$$
\begin{array}{ccc}
\check{R}^{\iota_{2}}\left(\bar{u}^{(1)}\right)=R_{1}, & \check{R}^{\iota_{2}^{2}}\left(\bar{u}^{(2)}\right)=R_{4}, & \check{R}(u)=R_{7} \\
\check{R}^{\iota_{1}}\left(\bar{u}^{(1)}\right)=R_{2}, & \check{R}^{\iota_{1} \iota_{2}}\left(\bar{u}^{(2)}\right)=R_{5}, & \check{R}^{\iota_{1} \iota_{2}^{2}}(u)=R_{8}, \\
\check{R}^{\iota_{1}^{2} \iota_{2}^{2}}\left(\bar{u}^{(1)}\right)=R_{3}, & \check{R}^{\iota_{1}^{2}}\left(\bar{u}^{(2)}\right)=R_{6}, & \check{R}^{\iota_{1}^{2} \iota_{2}}(u)=R_{9},
\end{array}
$$

with the corresponding numerations for the $a(0), \quad b(0)$ parameters(the parameter $c$ is the 
same for all of $R$-matrices and equal to $c=\cos \lambda$.

$$
\begin{array}{lll}
a_{1}=\sin \left(\epsilon^{2} \theta+\lambda\right) & a_{2}=\sin (\theta+\lambda) & a_{3}=\sin (\epsilon \theta+\lambda), \\
b_{1}=\sin \left(\epsilon^{2} \theta\right) & b_{2}=\sin (\theta) & b_{3}=\sin (\epsilon \theta), \\
a_{4}=\sin (-\theta+\lambda) & a_{5}=\sin (-\epsilon \theta+\lambda) & a_{6}=\sin \left(-\epsilon^{2} \theta+\lambda\right), \\
b_{4}=\sin (-\theta) & b_{5}=\sin (-\epsilon \theta) & b_{6}=\sin \left(-\epsilon^{2} \theta\right), \\
a_{7}=\sin (\lambda) & a_{8}=\sin (\lambda) & a_{9}=\sin (\lambda), \\
b_{7}=0 & b_{8}=0 & b_{9}=0
\end{array}
$$

and for their derivatives $a^{\prime}(0), b^{\prime}(0)$

$$
\begin{array}{lll}
a_{1}^{\prime}=\cos \left(\epsilon^{2} \theta+\lambda\right) & a_{2}^{\prime}=\epsilon \cos (\theta+\lambda) & a_{3}^{\prime}=\epsilon^{2} \cos (\epsilon \theta+\lambda), \\
b_{1}^{\prime}=\cos \left(\epsilon^{2} \theta\right) & b_{2}^{\prime}=\epsilon \cos (\theta) & b_{3}^{\prime}=\epsilon^{2} \cos (\epsilon \theta), \\
a_{4}^{\prime}=\cos (-\theta+\lambda) & a_{5}^{\prime}=\epsilon \cos (-\epsilon \theta+\lambda) & a_{6}^{\prime}=\epsilon^{2} \cos \left(-\epsilon^{2} \theta+\lambda\right), \\
b_{4}^{\prime}=\cos (-\theta) & b_{5}^{\prime}=\epsilon \cos (-\epsilon \theta) & b_{6}^{\prime}=\epsilon^{2} \cos \left(-\epsilon^{2} \theta\right) .
\end{array}
$$

The Hamiltonian (3.7-3.8) can have a following simple interpretation. We are taking the logarithmic derivatives of the nine $R$-matrices of the constituent block, but instead of ordinary sum we should take the braided sums of this terms. The meaning is clear from Figure 4 , where the square box represents $H$ and where crossings of lines represent $R$-matrices.
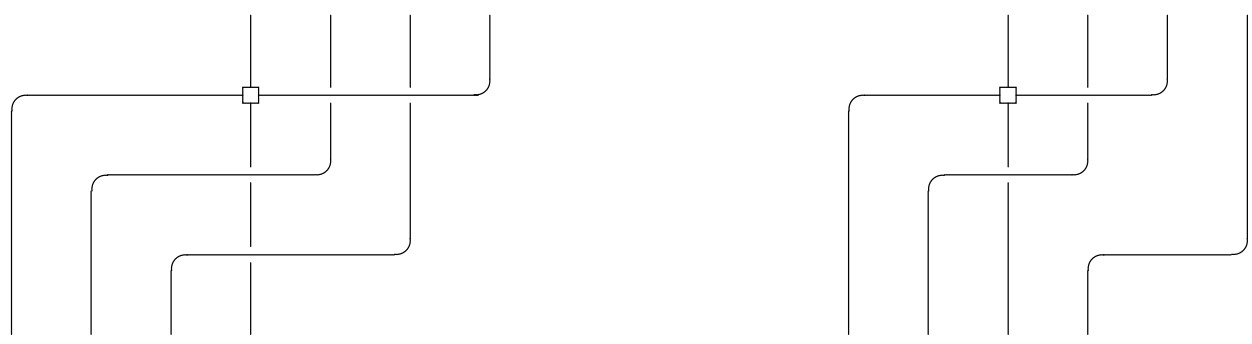

Figure 4: Some terms of the Hamiltonian

First one should make the permutations of neighbouring nodes with the corresponding $R^{\iota_{1}^{k} \iota_{2}^{p}}(0)$ matrix and after the interaction via the local Hamiltonian permute them back to their original order with the inverse matrices $\left(R^{\iota_{1}^{k} \iota_{2}^{p}}\right)^{-1}(0)$. This interpretation is interesting from the mathematical point of view and would need further investigation. Nevertheless it looks necessary to write down explicitly the expression for the Hamiltonian in terms of fermionic creation-annihilation operators.

In order to make the calculations it appeared to be convenient to pass from ordinary matrix (2.17) or operator $2.20-2.21)$ expressions of the $R$-matrix to the so called coherent 
state basis representation. Let us introduce the fermionic coherent states according to articles [21] and express the $R$-matrix in these terms as it is done in [2, 3].

$$
\left|\psi_{2 j}\right\rangle=e^{\psi_{2 j} c_{2 j}^{+}}|0\rangle, \quad\left\langle\bar{\psi}_{2 j}\right|=\langle 0| e^{c_{2 j} \bar{\psi}_{2 j}}
$$

for the even sites of the chain and

$$
\left|\bar{\psi}_{2 j+1}\right\rangle=\left(c_{2 j+1}^{+}-\bar{\psi}_{2 j+1}\right)|0\rangle, \quad\left\langle\psi_{2 j+1}\right|=\langle 0|\left(c_{2 j+1}+\psi_{2 j+1}\right)
$$

for the odd sites.

These states are designed as an eigenstates of creation-annihilation operators of fermions $c_{j}^{+}, \quad c_{j}$ with eigenvalues $\psi_{j}$ and $\bar{\psi}_{j}$

$$
\begin{array}{cl}
c_{2 j}\left|\psi_{2 j}\right\rangle=-\psi_{2 j}\left|\psi_{2 j}\right\rangle & ,\left\langle\bar{\psi}_{2 j}\right| c_{2 j}^{+}=-\left\langle\bar{\psi}_{2 j}\right| \bar{\psi}_{2 j}, \\
c_{2 j+1}^{+}\left|\bar{\psi}_{2 j+1}\right\rangle=\bar{\psi}_{2 j+1}\left|\bar{\psi}_{2 j+1}\right\rangle & , \quad\left\langle\psi_{2 j+1}\right| c_{2 j+1}=-\left\langle\psi_{2 j+1}\right| \psi_{2 j+1} .
\end{array}
$$

It is easy to calculate the scalar product of this states

$$
\begin{array}{r}
\left\langle\bar{\psi}_{2 j} \mid \psi_{2 j}\right\rangle=e^{\bar{\psi}_{2 j} \psi_{2 j}}, \\
\left\langle\psi_{2 j+1} \mid \bar{\psi}_{2 j+1}\right\rangle=e^{\bar{\psi}_{2 j+1} \psi_{2 j+1}}
\end{array}
$$

and find the completeness relations

$$
\begin{aligned}
\int d \bar{\psi}_{2 j} d \psi_{2 j}\left|\psi_{2 j}\right\rangle\left\langle\bar{\psi}_{2 j}\right| e^{\psi_{2 j} \bar{\psi}_{2 j}} & =1 \\
\int d \bar{\psi}_{2 j+1} d \psi_{2 j+1}\left|\bar{\psi}_{2 j+1}\right\rangle\left\langle\psi_{2 j+1}\right| e^{\psi_{2 j+1} \bar{\psi}_{2 j+1}} & =1 .
\end{aligned}
$$

Now let us pass to the coherent basis in the spaces of states $\prod_{j} V_{j, \sigma}$ of the chain and calculate the matrix elements of the $R_{2 j, 2 j \pm 1}$-operators between the initial $\left|\psi_{2 j}\right\rangle, \quad\left|\bar{\psi}_{2 j \pm 1}\right\rangle$ and final $\left\langle\bar{\psi}_{2 j}^{\prime}\right|,\left\langle\psi_{2 j \pm 1}^{\prime}\right|$ states. Using the properties of coherent states it is easy to find from the formula (2.20), that

$$
\begin{aligned}
&(R)_{\psi_{2 j}, \bar{\psi}_{2 j \pm 1}}^{\bar{\psi}_{2 j}^{\prime}, \psi_{2 j 1}^{\prime}}(u)=\left\langle\psi_{2 j \pm 1}^{\prime}, \bar{\psi}_{2 j}^{\prime}\left|\left(\check{R}_{k}\right)_{2 j, 2 j \pm 1}\right| \psi_{2 j}, \bar{\psi}_{2 j \pm 1}\right\rangle \\
&=e^{\left[a(u) \bar{\psi}_{2 j}^{\prime} \psi_{2 j}+a(u) \bar{\psi}_{2 j \pm 1} \psi_{2 j \pm 1}^{\prime}-b(u) \bar{\psi}_{2 j \pm 1} \psi_{2 j}+b(u) \bar{\psi}_{2 j}^{\prime} \psi_{2 j \pm 1}^{\prime}-\delta \bar{\psi}_{2 j}^{\prime} \psi_{2 j} \bar{\psi}_{2 j \pm 1} \psi_{2 j \pm 1}^{\prime}\right]} \\
& k=1,2 \ldots, 9 .
\end{aligned}
$$

where $\delta=2 a(u) b(u) \Delta$ and $\Delta=\cos (\lambda)$ is the models anisotropy parameter.

By use of these coherent states we can replace all the ordinary matrix multiplications in the expressions (3.7 3.8) of the Hamiltonian by the Grassmann variable integration with the measure

$$
\int \cdots e^{\psi_{i} \bar{\psi}_{i}} d \bar{\psi}_{i} d \psi_{i}
$$


as it is dictated by the formulae (3.16). Then the calculation of integrals over Grassmann variables simply reduces to the all possible Wilson-Polyakov's contour integral countings defined on a graph connected to the terms in the expression (3.7 3.7) by use of identification of the Figure 2 with the $R$-matrix.

The expression we have obtained for the $\mathcal{H}_{01234}^{(1)}$ is the following

$$
\begin{aligned}
\mathcal{H}_{01234}^{(1)} & =c_{2}^{+} c_{1}\left(t_{21}+f_{21} n_{3}\right)+c_{1}^{+} c_{2}\left(t_{12}+f_{12} n_{3}\right)+c_{2}^{+} c_{3}\left(t_{23}+f_{23} n_{1}\right)+c_{3}^{+} c_{2}\left(t_{32}+f_{32} n_{1}\right) \\
& +c_{3}^{+} c_{1}\left(t_{31}+f_{31} n_{2}\right)+c_{1}^{+} c_{3}\left(t_{13}+f_{13} n_{2}\right)+d_{11} n_{1}+d_{22} n_{2}+d_{33} n_{3}-d_{12} n_{1} n_{2} \\
& +d_{13} n_{1} n_{3}-d_{23} n_{2} n_{3}+d_{123} n_{1} n_{2} n_{3}
\end{aligned}
$$

where the coefficients $t_{i j}, f_{i j}, d_{i j}, i, j=1,2,3$ and $d_{123}$ are written down in the Appendix.

The expression for the second part of Hamiltonian $\mathcal{H}_{01234}^{(2)}$ which consist of derivatives of the $R_{7}(u), R_{8}(u), R_{9}(u)$ terms in the transfer matrix can be summarised as follows.

$$
\begin{aligned}
\mathcal{H}_{01234}^{(2)} & =H_{7,0}^{0} \cdot \mathcal{S}_{1,1}^{0} \cdot \mathcal{S}_{4,2}^{0}+H_{7,0}^{\mu} \cdot\left(\mathcal{S}_{1,1}\right)_{\mu}^{n u} \cdot\left(\mathcal{S}_{4,2}\right)_{\nu}^{\sigma} \cdot X_{3, \sigma} \\
& +\delta_{7}^{\prime}\left(\mathcal{S}_{4,0}^{T}\right)^{0} \cdot \overline{\mathcal{S}}_{3,1}^{0}+X_{-1}^{\sigma} \cdot\left(\mathcal{S}_{4,0}^{T}\right)_{\sigma}^{\mu} \cdot\left(H_{8}\right)_{\mu}^{\nu} \cdot\left(\overline{\mathcal{S}}_{3,1}\right)_{\nu}^{\rho} \cdot X_{2, \rho} \\
& +\left(\overline{\mathcal{S}}_{3,2}^{T}\right)^{0} \cdot\left(\overline{\mathcal{S}}_{6,3}^{T}\right)^{0} \cdot H_{9,4}^{0}+X_{1}^{\sigma} \cdot\left(\overline{\mathcal{S}}_{3,2}^{T}\right)_{\sigma}^{\mu} \cdot\left(\overline{\mathcal{S}}_{6,3}^{T}\right)_{\mu}^{\nu} \cdot\left(H_{9,4}\right)_{\nu},
\end{aligned}
$$

where vector indices $\mu, \nu, \sigma=+,-, 3$ and can be taken up and down by the usual metric tensor $g_{\mu \nu}=\left(\begin{array}{lll}0 & 2 & 0 \\ 2 & 0 & 0 \\ 0 & 0 & 1\end{array}\right)$, upper script $T$ means transposition.

In the expression (3.20) other notations are defined as

$$
\begin{array}{rlr}
H_{r, i}^{0} & =a_{r}^{\prime}-\left(\delta_{r}^{\prime}-a_{r}^{\prime}\right) c_{i}^{+} c_{i}, \\
H_{r, i}^{\mu} & =\left(b_{r}^{\prime} c_{i}^{+}, b_{r}^{\prime} c_{i}^{\prime} a_{r}^{\prime}-\left(\delta_{r}^{\prime}+a_{r}^{\prime}\right) c_{i}^{+} c_{i}\right), \\
\left(H_{r}\right)_{\mu}^{\nu} & =\left(\begin{array}{ccc}
2 b_{r}^{\prime} & 0 & 0 \\
0 & -2 b_{r}^{\prime} & 0 \\
0 & 0 & \delta_{r}^{\prime}
\end{array}\right), \\
X_{i}^{\mu} & =\left(c_{i}, c_{i}^{+}, 1-2 n_{i}\right) &
\end{array}
$$

and

$$
\begin{aligned}
& \mathcal{S}_{r, i}^{0}=2 a_{r} \bar{a}_{r} e^{c_{i}^{+} c_{i}}, \quad r=7,8,9 \\
& \left(\mathcal{S}_{r, i}\right)_{\mu}^{\nu}=\left(\begin{array}{ccc}
-2 b_{r} \bar{a}_{r} e^{\frac{a_{r}}{\bar{a}_{r}} c_{i}^{+} c_{i}} & 0 & \sqrt{2}\left(\bar{a}_{r}-a_{r}\right) c_{i} \\
0 & -2 b_{r} \bar{a}_{r} e^{\frac{\bar{a}_{r}}{a_{r}} c_{i}^{+} c_{i}} & \sqrt{2}\left(\bar{a}_{r}-a_{r}\right) c_{i}^{+} \\
-2 \sqrt{2} b_{r} c_{i}^{+} & -2 \sqrt{2} b_{r} c_{i} & 2 b_{r}^{2} e^{c_{i}^{+} c_{i}}
\end{array}\right)
\end{aligned}
$$

where subscript $i$ enumerates the lattice site. In the formulae (3.22) $\bar{a}_{1}=a_{6}, \bar{a}_{2}=$ $a_{4}, \bar{a}_{3}=a_{5}, \bar{a}_{4}=a_{2}, \bar{a}_{5}=a_{3}, \bar{a}_{6}=a_{1}$. 


\section{Algebra}

The underlying algebra of this model is defined by generators $L^{\iota^{p}}$ and the following set of RLL relations derived from the set of coupled Yang-Baxter equations following [18]

$$
\begin{aligned}
& R_{12}^{\iota^{p}} L_{1}^{ \pm \iota^{p+1}} L_{2}^{ \pm \iota^{p}}=L_{2}^{ \pm \iota^{\iota^{p+1}}} L_{1}^{ \pm \iota^{p}} R_{12}^{\iota^{p+1}} \\
& R_{12}^{\iota^{p}} L_{1}^{+\iota^{p+1}} L_{2}^{-\iota^{p}}=L_{2}^{-\iota^{\iota^{p+1}}} L_{1}^{+\iota^{p}} R_{12}^{\iota^{p+1}}
\end{aligned}
$$

(with again $\iota \equiv \iota_{1}$ ). Writing

$$
L^{+\iota^{p}}=\left(\begin{array}{cc}
K_{+1^{\iota^{p}}} & 0 \\
E^{\iota^{p}} & K_{+2}^{\iota^{p}}
\end{array}\right), \quad L^{-\iota^{p}}=\left(\begin{array}{cc}
K_{-1}^{\iota^{p}} & \iota^{p} F \\
0 & K_{-2}^{\iota^{p}}
\end{array}\right)
$$

these relations become

$$
\begin{aligned}
& K_{+1}^{\iota_{1}^{p+1}} K_{-1}^{\iota_{1}^{p}}=K_{-1}^{\iota_{1}^{p+1}} K_{+1}^{\iota_{1}^{p}} \\
& K_{+2}^{\iota_{1}^{p+1}} K_{-2}^{\iota_{1}^{p}}=K_{-2}^{\iota_{1}^{p+1}} K_{+2}^{\iota_{1}^{p}} \\
& K_{+1}^{\iota_{1}^{p+1}} K_{+2}^{\iota_{1}^{p}}=\epsilon K_{+2}^{\iota_{1}^{p+1}} K_{+1}^{\iota_{1}^{p}} \\
& K_{-1}^{\iota_{1}^{p+1}} K_{-2}^{\iota_{1}^{p}}=\epsilon K_{-2}^{\iota_{1}^{p+1}} K_{-1}^{\iota_{1}^{p}} \\
& K_{+1}^{\iota_{1}^{p+1}} K_{-2}^{\iota_{1}^{p}}=\epsilon K_{-2}^{\iota_{1}^{p+1}} K_{+1}^{\iota_{1}^{p}} \\
& K_{+2}^{\iota_{1}^{p+1}} K_{-1}^{\iota_{1}^{p}}=\epsilon^{-1} K_{-1}^{\iota_{1}^{p+1}} K_{+2}^{\iota_{1}^{p}} \\
& K_{+1}^{\iota_{1}^{p+1}} E^{\iota_{1}^{p}}=\epsilon^{-p} q E^{\iota_{1}^{p+1}} K_{+1}^{\iota_{1}^{p}} \\
& K_{+1}^{\iota_{1}^{p+1}} F^{\iota_{1}^{p}}=\epsilon^{p+1} q^{-1} F^{\iota_{1}^{p+1}} K_{+1}^{\iota_{1}^{p}} \\
& K_{-1}^{\iota_{1}^{p+1}} E^{\iota_{1}^{p}}=\epsilon^{-p} q^{-1} E^{\iota_{1}^{p+1}} K_{-1}^{\iota_{1}^{p}} \\
& K_{-1}^{\iota_{1}^{p+1}} F^{\iota_{1}^{p}}=\epsilon^{p+1} q F^{\iota_{1}^{p+1}} K_{-1}^{\iota_{1}^{p}} \\
& K_{+2}^{\iota_{1}^{p+1}} E^{\iota_{1}^{p}}=\epsilon^{-p-1} q^{-1} E^{\iota_{1}^{p+1}} K_{+2}^{\iota_{1}^{p}} \\
& K_{+2}^{\iota_{1}^{p+1}} F^{\iota_{1}^{p}}=\epsilon^{p} q F^{\iota_{1}^{p+1}} K_{+2}^{\iota_{1}^{p}} \\
& K_{-2}^{\iota_{1}^{p+1}} E^{\iota_{1}^{p}}=\epsilon^{-p-1} q E^{\iota_{1}^{p+1}} K_{-2}^{\iota_{1}^{p}} \\
& K_{-2}^{\iota_{1}^{p+1}} F^{\iota_{1}^{p}}=\epsilon^{p} q^{-1} F^{\iota_{1}^{p+1}} K_{-2}^{\iota_{1}^{p}} \\
& \epsilon^{-p} E^{\iota_{1}^{p+1}} F^{\iota_{1}^{p}}-\epsilon^{p+1} F^{\iota_{1}^{p+1}} E^{\iota_{1}^{p}}=\left(q-q^{-1}\right)\left(K_{-1}^{\iota_{1}^{p+1}} K_{+2}^{\iota_{1}^{p}}-K_{+1}^{\iota_{1}^{p+1}} K_{-2}^{\iota_{1}^{p}}\right)
\end{aligned}
$$

We further define

$$
\begin{aligned}
& e^{\iota^{p}} \equiv E^{\iota_{1}^{p-1}}\left(K_{+1}^{\iota_{1}^{p-1}}\right)^{-1}=\epsilon^{-p+1} q\left(K_{+1}^{\iota_{1}^{p}}\right)^{-1} E^{\iota_{1}^{p}} \\
& f^{\iota^{p}} \equiv F^{\iota_{1}^{p-1}}\left(K_{-1}^{\iota_{1}^{p-1}}\right)^{-1}=\epsilon^{p} q\left(K_{-1}^{\iota_{1}^{p}}\right)^{-1} F^{\iota_{1}^{p}} \\
& k_{-1}^{\iota^{p}} \equiv K_{-1}^{\iota_{1}^{p-1}}\left(K_{+1}^{\iota_{1}^{p-1}}\right)^{-1}=\left(K_{+1}^{\iota_{1}^{p}}\right)^{-1} K_{-1}^{\iota_{1}^{p}}
\end{aligned}
$$




$$
\begin{aligned}
& k_{2}^{\iota^{p}} \equiv K_{+2}^{\iota_{1}^{p-1}}\left(K_{+1}^{\iota_{1}^{p-1}}\right)^{-1}=\epsilon\left(K_{+1}^{\iota_{1}^{p}}\right)^{-1} K_{+2}^{\iota_{1}^{p}} \\
& k_{-2}^{\iota^{p}} \equiv K_{-2}^{\iota_{1}^{p-1}}\left(K_{+1}^{\iota_{1}^{p-1}}\right)^{-1}=\epsilon\left(K_{+1}^{\iota_{1}^{p}}\right)^{-1} K_{-2}^{\iota_{1}^{p}}
\end{aligned}
$$

These operators fulfil the relations

$$
\begin{aligned}
k_{-1}^{\iota^{p}} e^{\iota^{p^{\prime}}}=\delta_{p, p^{\prime}} q^{-2} e^{\iota^{p}} k_{-1}^{\iota^{p}} & k_{-1}^{\iota^{p}} f^{\iota^{p^{\prime}}}=\delta_{p, p^{\prime}} q^{2} f^{\iota^{p}} k_{-1}^{\iota^{p}} \\
k_{2}^{\iota^{p}} e^{\iota^{p^{\prime}}}=\delta_{p, p^{\prime}} q^{-2} e^{\iota^{p}} k_{2}^{\iota^{p}} & k_{2}^{\iota^{p}} f^{\iota^{p^{\prime}}}=\delta_{p, p^{\prime}} q^{2} f^{\iota^{p}} k_{2}^{\iota^{p}} \\
k_{-2}^{\iota^{p}} e^{\iota^{p^{\prime}}}=\delta_{p, p^{\prime}} e^{\iota^{p}} k_{-2}^{\iota^{p}} & k_{-2}^{\iota^{p}} f^{\iota^{p^{\prime}}}=\delta_{p, p^{\prime}} f^{\iota^{p}} k_{-2}^{\iota^{p}} \\
& {\left[e^{\iota^{p}}, f^{\iota^{p^{\prime}}}\right]=\delta_{p, p^{\prime}}\left(q^{2}-1\right)\left(k_{2}^{\iota^{p}}-k_{-2}^{\iota^{p}} k_{-1}^{\iota^{p}}\right) }
\end{aligned}
$$

and, together with $K_{1}^{\iota^{p}}$

$$
\begin{aligned}
& K_{1}^{\iota^{p}} e^{\iota^{p^{\prime}}}=\delta_{p, p^{\prime}} \epsilon^{-p+1} q e^{\iota^{p+1}} K_{1}^{\iota^{p}} \\
& K_{1}^{\iota^{p}} f^{\iota^{p^{\prime}}}=\delta_{p, p^{\prime}} \epsilon^{p} q^{-1} f^{\iota^{p+1}} K_{1}^{\iota^{p}} \\
& K_{1}^{\iota^{p}} k_{-1}^{\iota^{p^{\prime}}}=\delta_{p, p^{\prime}} k_{-1}^{\iota^{p+1}} K_{1}^{\iota^{p}} \\
& K_{1}^{\iota^{p}} k_{2}^{\iota^{p^{\prime}}}=\delta_{p, p^{\prime}} \epsilon k_{2}^{\iota^{p+1}} K_{1}^{\iota^{p}} \\
& K_{1}^{\iota^{p}} k_{-2}^{\iota^{p^{\prime}}}=\delta_{p, p^{\prime}} \in k_{-2}^{\iota^{p+1}} K_{1}^{\iota^{p}}
\end{aligned}
$$

Proposition 4.1 The composite operators

$$
\begin{aligned}
& \mathcal{E} \equiv \frac{1}{q-q^{-1}} \sum_{p=0}^{P-1} \epsilon^{-\frac{1}{2} p(p-3)} q^{p} e^{\iota^{p}}, \quad \mathcal{F} \equiv \frac{1}{q-q^{-1}} \sum_{p=0}^{P-1} \epsilon^{\frac{1}{2} p(p-1)} q^{-p-1} f^{\iota^{p}} \\
& \mathcal{K}_{-1} \equiv \sum_{p=0}^{P-1} k_{-1}^{\iota^{p}}, \quad \mathcal{K}_{2} \equiv \sum_{p=0}^{P-1} \epsilon^{p} k_{2}^{\iota^{p}}, \quad \mathcal{K}_{-2} \equiv \sum_{p=0}^{P-1} \epsilon^{p} k_{-2}^{\iota^{p}}
\end{aligned}
$$

satisfy relations isomorphic to those of $\mathcal{U}_{q}(g l(2))$, i.e.

$$
\begin{array}{lc}
\mathcal{K}_{-1} \mathcal{E}=q^{-2} \mathcal{E} \mathcal{K}_{-1} & \mathcal{K}_{-1} \mathcal{F}=q^{2} \mathcal{F} \mathcal{K}_{-1} \\
\mathcal{K}_{2} \mathcal{E}=q^{-2} \mathcal{E} \mathcal{K}_{2} & \mathcal{K}_{2} \mathcal{F}=q^{2} \mathcal{F} \mathcal{K}_{2} \\
\mathcal{K}_{-2} \mathcal{E}=\mathcal{E} \mathcal{K}_{-2} & \mathcal{K}_{-2} \mathcal{F}=\mathcal{F} \mathcal{K}_{-2} \\
& \\
&
\end{array}
$$

$\left(\mathcal{K}_{-2}\right.$ and $\mathcal{K}_{2} \mathcal{K}_{-1}$ being central. $)$ 
Proof: By direct check.

The supplementary operator

$$
\ell=\sum_{p=0}^{P-1} \coprod_{r=0, . ., P-1} K_{1}^{(p+r)}
$$

(with by convention $K_{1}^{(a+P)} \equiv K_{1}^{(a)}$ ) is central for odd $P$ and it acts as " $i^{h}$ " for even $P$, i.e. it anticommutes with $\mathcal{E}, \mathcal{F}$ and commutes with $\mathcal{K}_{-1}, \mathcal{K}_{ \pm 2}$. This operator corresponds for even $P$ to the existence of another Cartan generator related to a second deformation parameter $q^{\prime}=i$, as observed in [7]. Most general multiparameter deformations were considered in 222].

The operators $\mathcal{E}, \mathcal{F}$ and $\mathcal{K}_{-1}, \mathcal{K}_{ \pm 2}$ are more clearly understood when written in a matrix form reflecting the $\mathbb{Z}_{P}$-grading

$$
\mathcal{E}=\left(\begin{array}{cccc}
e^{(0)} & 0 & \cdots & \\
0 & e^{(1)} & & \vdots \\
& & \ddots & \\
& \cdots & & e^{(P-1)}
\end{array}\right) \quad \mathcal{F}=\left(\begin{array}{cccc}
f^{(0)} & 0 & \cdots & \\
0 & f^{(1)} & & \vdots \\
& & \ddots & \\
& \ldots & & f^{(P-1)}
\end{array}\right)
$$

and similarly for $\mathcal{K}_{-1}, \mathcal{K}_{ \pm 2}$.

We finally need to introduce the operators $\overline{\mathcal{K}}_{1}$ and $\mathcal{X}$ defined by

$$
\overline{\mathcal{K}}_{1} \equiv \sum_{p=0}^{P-2} K_{1}^{\iota^{p}}+\left(K_{1}\right)^{-1}\left(K_{1}^{\iota}\right)^{-1} \ldots\left(K_{1}^{\iota^{P-2}}\right)^{-1}
$$

and

$$
\left.\mathcal{X} \equiv \sum_{p=0}^{P-1} \epsilon^{p} \mathrm{Id}\right|_{V_{p}}
$$

i.e., in matrix form:

$$
\overline{\mathcal{K}}_{1}=\left(\begin{array}{cccc}
0 & 0 & \cdots & \prod_{p=0}^{P-2} K_{1}^{(p)^{-1}} \\
K_{1}^{(0)} & 0 & & \vdots \\
0 & K_{1}^{(1)} & & \vdots \\
& & \ddots & \\
& \cdots & K_{1}^{(P-2)} & 0
\end{array}\right) \quad \mathcal{X}=\left(\begin{array}{cccc}
1 & 0 & \ldots & \\
0 & \epsilon & & \vdots \\
& & \ddots & \\
& \ldots & & \epsilon^{P-1}
\end{array}\right)
$$

Proposition 4.2 The operators $E^{\iota^{p}}, F^{\iota^{p}}, K_{ \pm 1,2}^{\iota^{p}}$ generate an algebra isomorphic to $\mathcal{W} \otimes$ $\mathcal{U}_{q}(g l(2))$, where $\mathcal{W}$ is a $\epsilon$-Weyl algebra, i.e.

$$
\overline{\mathcal{K}}_{1}^{P}=\mathcal{X}^{P}=1 \quad \mathcal{X} \overline{\mathcal{K}}_{1}=\epsilon \overline{\mathcal{K}}_{1} \mathcal{X}
$$

$\left(\mathcal{U}_{q}(g l(2))\right.$ being understood with the supplementary operator $\ell$ (4.2J)). 
Proof: First one checks that

$$
\begin{array}{ll}
{\left[\overline{\mathcal{K}}_{1}, \mathcal{E}\right]=0} & {\left[\overline{\mathcal{K}}_{1}, \mathcal{K}_{ \pm 2}\right]=0} \\
{\left[\overline{\mathcal{K}}_{1}, \mathcal{F}\right]=0} & {\left[\overline{\mathcal{K}}_{1}, \mathcal{K}_{-1}\right]=0 .}
\end{array}
$$

$\mathcal{X}$ also obviously commutes with $\mathcal{E}, \mathcal{F}, \mathcal{K}_{ \pm 2}$ and $\mathcal{K}_{-1}$.

Then it remains to write all the generators $E^{\iota^{p}}, F^{\iota^{p}}, K_{ \pm 1,2}^{\iota^{p}}$, in terms of $\mathcal{E}, \mathcal{F}, \mathcal{K}_{ \pm 2}, \ell, \overline{\mathcal{K}}_{1}$ and $\mathcal{X}$, which can be done using polynomials of $\mathcal{X}$ for projections on $V_{p}$ and then inverting the relations (4.7)-(4.11).

\section{$5 \quad$ Algebraic Bethe Ansatz $(A B A)$ solution of the model}

The Algebraic Bethe Ansatz $(A B A)$ technique, called also Quantum Inverse Scattering Method (QISM), was essentially developed in the works of Baxter [8] and of Leningrad group [9].

In order to carry out $A B A$ it is convenient to work in the conventional (not braid) formalism and use the $Y B E$ for $R$-operators in the form of formulas (2.8) and (2.21). Let us first define the $L$-matrix as

$$
\begin{aligned}
\left(L_{j}^{\iota_{1}^{p}}(u)\right)_{a^{\prime}}^{a} & ={ }_{i}\left\langle a\left|R_{i j}^{\iota_{1}^{p}}\right| a^{\prime}\right\rangle_{i}=(-1)^{p\left(a^{\prime}\right) p\left(b^{\prime}\right)}\left(R_{i j}^{\iota_{1}^{p}}(u)\right)_{a^{\prime} b^{\prime}}^{a b} X_{j b}^{b^{\prime}} \\
& =\left(\begin{array}{ll}
a(u)\left(1-n_{j}\right)+\epsilon^{-p} b(u) n_{j} & c(u) c_{j}^{+} \\
c(u) c_{j} & -a(u) n_{j}+\epsilon^{p} b(u)\left(1-n_{j}\right)
\end{array}\right),
\end{aligned}
$$

which is a matrix in the horizontal auxiliary space with operator value entries acting on quantum space $V_{j}$. The matrix elements between auxiliary states $\left|a^{\prime}\right\rangle$ and $\langle a|$ of the monodromy operators $T_{s}(\lambda, u)$ defined in (2.7)

$$
\left(T_{s}(u)\right)_{a}^{a^{\prime}}=\left\langle a^{\prime}\left|T_{s}(u)\right| a\right\rangle=\left(\begin{array}{cc}
A_{s}(u) & B_{s}(u) \\
C_{s}(u) & D_{s}(u)
\end{array}\right), \quad s=0,1, \ldots P-1
$$

can be expressed as a product of the $L$-matrices as follows

$$
\begin{array}{r}
T_{0}(u)=\prod_{j=0}^{L / P}\left(L_{P j}(u) L_{P j+1}^{\iota_{2}}(\bar{u}) \cdots L_{P j+P-1}^{\iota_{2}^{P-1}}\left(\bar{u}^{(P-1)}\right)\right), \\
T_{1}(u)=\prod_{j=0}^{L / P}\left(L_{P j}^{\iota_{1}}(\bar{u}) L_{P j+1}^{\iota_{1} \iota_{2}}\left(\bar{u}^{(2)}\right) \cdots L_{P j+P-1}^{\iota_{1} \ell_{2}^{P-1}}\left(\bar{u}^{(P)}\right)\right), \\
T_{p}(u)=\prod_{j=0}^{L / P}\left(L_{P j}^{\iota_{1}^{p}}\left(\bar{u}^{(p)}\right) L_{P j+1}^{L_{1}^{p} \iota_{2}}\left(\bar{u}^{(p+1)}\right) \cdots L_{P j+P-1}^{\iota_{1}^{p} P_{2}^{P-1}}\left(\bar{u}^{(p+P-1)}\right)\right), \\
p=0,1 \ldots, P-1 .
\end{array}
$$


By use of equations (2.8) one can obtain the graded $Y B E$ for the monodromy matrices $T_{p}, \quad p=0,1, \ldots P-1$ as follows

$$
\begin{aligned}
& (-1)^{\left(P\left(b^{\prime}\right)+P\left(b^{\prime \prime}\right)\right) P\left(a^{\prime \prime}\right)}(R)_{a^{\prime} b^{\prime}}^{a b}\left(\bar{v}^{(1)}-u\right)\left(T_{0}\right)_{a^{\prime \prime}}^{a^{\prime}}(u)\left(T_{1}\right)_{b^{\prime \prime}}^{b^{\prime}}(v) \\
& \quad=(-1)^{P\left(b^{\prime}\right)\left(P\left(a^{\prime}\right)+P(a)\right)}\left(T_{0}\right)_{b^{\prime}}^{b}(v)\left(T_{1}\right)_{a^{\prime}}^{a}(u)(R)_{a^{\prime \prime} b^{\prime \prime}}^{a^{\prime} b^{\prime}}\left(\bar{v}^{(1)}-u\right) \\
& \quad \vdots \\
& (-1)^{\left(P\left(b^{\prime}\right)+P\left(b^{\prime \prime}\right)\right) P\left(a^{\prime \prime}\right)}\left(R^{\iota_{1}^{p}}\right)_{a^{\prime} b^{\prime}}^{a b}\left(\bar{v}^{(p+1)}-\bar{u}^{(p)}\right)\left(T_{p}\right)_{a^{\prime \prime}}^{a^{\prime}}(u)\left(T_{p+1}\right)_{b^{\prime \prime}}^{b^{\prime}}(v) \\
& =(-1)^{P\left(b^{\prime}\right)\left(P\left(a^{\prime}\right)+P(a)\right)}\left(T_{p}\right)_{b^{\prime}}^{b}(v)\left(T_{p+1}\right)_{a^{\prime}}^{a}(u)\left(R^{\iota^{p}}\right)_{a^{\prime \prime} b^{\prime \prime}}^{a^{\prime} b^{\prime}}\left(\bar{v}^{(p+1)}-\bar{u}^{(p)}\right) .
\end{aligned}
$$

Now, following the procedure of $A B A$, let us define empty fermionic state

$$
|\Omega\rangle=\prod_{i=1}^{L}|0\rangle_{i}
$$

as a test vacuum of the model and demonstrate that it is the eigenstate of the transfer matrix $\tau(\lambda, u)=\prod_{p=0}^{P-1} \tau_{p}(\lambda, u)=\operatorname{Tr} T(\lambda, u)$.

The expression (5.1) for $L$-matrix shows that its action on $|0\rangle_{i}$ produces upper triangular matrix, therefore the action of the monodromy matrix $\left(T_{p}\right)_{a}^{a^{\prime}}$ on $|\Omega\rangle$, defined by the formulas (5.2) and (5.3), will also have upper triangular form and can be calculated easily as

$$
\begin{aligned}
\left(T_{0}(u)\right)|\Omega\rangle & =\left(\begin{array}{ll}
{\left[\prod_{k=0}^{P-1} a^{\iota_{2}^{k}}\left(\bar{u}^{(k)}\right)\right]^{L / P}} & B_{0}(u) \\
0 & {\left[\prod_{k=0}^{P-1} b^{\iota_{2}^{k}}\left(\bar{u}^{(k)}\right)\right]^{L / P}}
\end{array}\right)|\Omega\rangle, \\
\vdots & \\
\left(T_{p}(u)\right)|\Omega\rangle & =\left(\begin{array}{ll}
{\left[\prod_{k=0}^{P-1} a^{\iota_{1}^{p} \iota_{2}^{k}}\left(\bar{u}^{(k)}\right)\right]^{L / P}} & B_{p}\left(\bar{u}^{(p)}\right) \\
0 & {\left[\prod_{k=0}^{P-1} b^{\iota_{1}^{p} \iota_{2}^{k}}\left(\bar{u}^{(k)}\right)\right]^{L / P}}
\end{array}\right)|\Omega\rangle .
\end{aligned}
$$

where

$$
\begin{array}{ll}
a^{\iota_{2}^{p}}(u)=a^{\iota_{1}^{p}}\left(\epsilon^{-p} u\right), & a^{\iota_{1}^{p}}(u)=a(u), \\
b^{\iota_{2}^{p}}(u)=b^{\iota_{1}^{p}}\left(\epsilon^{-p} u\right), & b^{\iota_{1}^{p}}(u)=\epsilon^{p} b(u), \\
c^{\iota_{2}^{p}}(u)=b^{\iota_{2}^{p}}\left(\epsilon^{-p} u\right), & c^{\iota_{1}^{p}}(u)=c(u) .
\end{array}
$$

Now it is obvious that $|\Omega\rangle$ is an eigenstate of $\tau(u)$ with eigenvalue

$$
\nu(u)=\prod_{p=0}^{P-1} \nu_{p}(u),
$$




$$
\begin{aligned}
\nu_{0}(u) & =\left[\prod_{k=0}^{P-1} a^{\iota_{2}^{k}}\left(\bar{u}^{(k)}\right)\right]^{L / P}+\left[\prod_{k=0}^{P-1} b^{\iota_{2}^{k}}\left(\bar{u}^{(k)}\right)\right]^{L / P}, \\
\vdots & \\
\nu_{p}(u) & =\left[\prod_{k=0}^{P-1} a^{\iota_{1}^{p} \iota_{2}^{k}}\left(\bar{u}^{(k)}\right)\right]^{L / P}+\left[\prod_{k=0}^{P-1} b^{\iota_{1}^{p} \iota_{2}^{k}}\left(\bar{u}^{(k)}\right)\right]^{L / P} .
\end{aligned}
$$

One can see from the expressions (5.6) that the operators $C_{p}(u)$ act on $|\Omega\rangle$ as the annihilation operators, while $B_{p}(u)$ act as the creation operators. That is why it is meaningful to look for states

$$
\left|v_{0}, v_{1}, \ldots v_{n}\right\rangle_{0}=B_{0}\left(v_{0}\right) B_{1}\left(v_{1}\right) \ldots B_{x}\left(v_{n}\right)|\Omega\rangle_{x}, \quad x=n(\bmod P),
$$

as an $n$-particle eigenstates of $\tau(u)$ with spectral parameters $v_{i}, i=0, \ldots n$. In order to check whether this is true we do not need to have an exact form of operators $B_{p}(u)$, we need only to know the algebra of operators $A_{p}(u), D_{p}(u)$ and $B_{p}(u)$, which can be found from the $Y B E$ (5.4) as follows

$$
\begin{gathered}
A_{0}(u) B_{1}(v)=+\frac{a(v-u)}{b(v-u)} B_{0}(v) A_{1}(u)-\frac{c(v-u)}{b(v-u)} B_{0}(u) A_{1}(v), \\
\vdots \\
A_{p}(u) B_{p+1}(v)=+\frac{a^{\iota_{1}^{p}}(v-u)}{b^{\iota^{p}}(v-u)} B_{p}(v) A_{p+1}(u)-\frac{c^{\iota_{1}^{p}}(v-u)}{b^{\iota_{1}^{p}}(v-u)} B_{p}(u), A_{p+1}(v) \\
p=0,1, \ldots P-1
\end{gathered}
$$

and

$$
\begin{gathered}
D_{0}(u) B_{1}(v)=+\frac{a(u-v)}{b(u-v)} B_{0}(v) D_{1}(u)+\frac{c(u-v)}{b(u-v)} B_{0}(u) D_{1}(v), \\
\vdots \\
D_{p}(u) B_{p+1}(v)=-\frac{a^{\iota_{1}^{p}}(u-v)}{b^{\iota_{1}^{p}}(u-v)} B_{p}(v) D_{p+1}(u)-\frac{c^{\iota_{1}^{p}}(u-v)}{b^{\iota_{1}^{p}}(u-v)} B_{p}(u) D_{p+1}(v), \\
p=0,1, \ldots P-1
\end{gathered}
$$

The first terms in the right hand side of equations (5.10) and (5.11) are so called "wanted" terms and they are producing the eigenvalues $\nu\left(u, v_{1}, v_{2}, . . v_{n}\right)$ of the state $\left|v_{1}, v_{2}, \ldots v_{n}\right\rangle_{0}$ as

$$
\nu\left(u, v_{1}, v_{2}, . . v_{n}\right)=\prod_{p=0}^{P-1} \nu_{p}\left(u, v_{1}, v_{2}, . . v_{n}\right),
$$




$$
\begin{aligned}
& \nu_{0}\left(u, v_{1}, \cdots, v_{n}\right)=\frac{1}{\prod_{i=1}^{n} b^{i_{1}^{i}}\left(v_{i}-u\right)} \cdot \\
& \qquad\left\{\prod_{i=1}^{n} a^{\iota_{1}^{i}}\left(v_{i}-u\right)\left[\prod_{k=0}^{P-1} a^{\iota_{2}^{k}}\left(\bar{u}^{(k)}\right)\right]^{L / P}+\prod_{i=1}^{n} a^{\iota_{1}^{i}}\left(u-v_{i}\right)\left[\prod_{k=0}^{P-1} b^{\iota_{2}^{k}}\left(\bar{u}^{(k)}\right)\right]^{L / P}\right\} \\
& \vdots \\
& \nu_{p}\left(u, v_{1}, \cdots, v_{n}\right)=\frac{1}{\prod_{i=1}^{n} b_{1}^{i+p}\left(v_{i}-\bar{u}^{(p)}\right)} \cdot \\
& \quad \cdot\left\{\prod_{i=1}^{n} a^{i_{1}^{i+p}}\left(v_{i}-\bar{u}^{(p)}\right)\left[\prod_{k=0}^{P-1} a^{\iota_{2}^{k+p}}\left(\bar{u}^{(k)}\right)\right]^{L / P}+\prod_{i=1}^{n} a^{\iota_{1}^{i+p}}\left(\bar{u}^{(p)}-v_{i}\right)\left[\prod_{k=0}^{P-1} b^{k_{2}^{k+p}}\left(\bar{u}^{(k)}\right)\right]^{L / P}\right\} \\
& p=0,1, \ldots P-1 .
\end{aligned}
$$

But in order the state $\left|v_{1}, v_{2}, \ldots v_{n}\right\rangle$ to be an eigenstate of $\tau(u)$ we need the cancellation of so called "unwanted" terms, produced by the second terms in the right hand side of equations (5.10) and (5.11). This gives us the restrictions on the spectral parameters $v_{1}, v_{2}, \ldots v_{n}$ in the form of Bethe Equations $(B E)$

$$
\left[\frac{\prod_{k=0}^{P-1} a^{\iota_{2}^{k}}\left(\bar{u}^{(k)}\right)}{\prod_{k=0}^{P-1} b^{\iota_{2}^{k}}\left(\bar{u}^{(k)}\right)}\right]^{N}=-\prod_{i \neq j}^{n} \frac{a^{\iota_{1}^{i}}\left(v_{j}-v_{i}\right)}{a^{\iota_{1}^{i}}\left(v_{i}-v_{j}\right)}, \quad j=1, \ldots n
$$

which completes the $A B A$ solution of our model.

\section{Appendix}

$$
\begin{aligned}
t_{12} & =\epsilon\left[b_{3}^{\prime}-\frac{b_{3} b_{6}^{2} a_{2}^{\prime}+b_{3}^{2} a_{1} b_{2}^{\prime}-a_{6} b_{2}^{\prime}-\delta_{2}^{\prime} a_{6} a_{1} b_{3}}{a_{1} a_{6} a_{3} a_{5}}+\frac{b_{1}^{\prime}}{a_{4}}\right. \\
& \left.-\frac{b_{5}^{\prime} b_{1} b_{4} a_{1}-a_{5}^{\prime} b_{1}}{a_{4} a_{6} a_{1}}-\frac{a_{6}^{\prime} b_{3}}{a_{3} a_{5}}\right], \\
t_{21} & =\epsilon^{-1}\left[b_{3}^{\prime}+\frac{b_{3} b_{6}^{2} a_{2}^{\prime}+b_{3}^{2} a_{6} b_{2}^{\prime}-a_{1} b_{2}^{\prime}-\delta_{2}^{\prime} a_{6} a_{1} b_{3}}{a_{1} a_{6} a_{3} a_{5}}+\frac{b_{1}^{\prime}}{a_{1}}\right. \\
& \left.-\frac{b_{5}^{\prime} b_{1} b_{4} a_{6}-a_{5}^{\prime} b_{1}}{a_{2} a_{6} a_{1}}+\frac{a_{6}^{\prime} b_{3}}{a_{3} a_{5}}\right], \\
t_{23} & =\epsilon\left[b_{4}^{\prime}+\frac{b_{6}\left(a_{2}^{\prime}-b_{2}^{\prime} b_{3} a_{6}\right)}{a_{3} a_{1} a_{6}}-\frac{a_{1}^{\prime} b_{4}}{a_{2} a_{4}}\right. \\
& \left.-\frac{b_{4}\left(b_{1}^{2} a_{5}^{\prime}+a_{6} b_{4} b_{5}^{\prime}\right)-a_{1}\left(b_{5}^{\prime}+\delta_{5}^{\prime} a_{6} b_{4}\right)}{a_{1} a_{6} a_{2} a_{4}}+\frac{b_{6}^{\prime}}{a_{3}}\right] \\
t_{32} & =\epsilon^{-1}\left[b_{4}^{\prime}-\frac{b_{6}\left(a_{2}^{\prime}+b_{2}^{\prime} b_{3} a_{1}\right)}{a_{5} a_{1} a_{6}}+\frac{a_{1}^{\prime} b_{4}}{a_{2} a_{4}}\right.
\end{aligned}
$$




$$
\begin{aligned}
& \left.+\frac{b_{4}\left(b_{1}^{2} a_{5}^{\prime}-a_{1} b_{4} b_{5}^{\prime}\right)+a_{6}\left(b_{5}^{\prime}-\delta_{5}^{\prime} a_{1} b_{4}\right)}{a_{1} a_{6} a_{2} a_{4}}+\frac{b_{6}^{\prime}}{a_{5}}\right], \\
& t_{13}=\epsilon^{-1}\left[-\frac{a_{2}^{\prime} b_{3} b_{6}+b_{2}^{\prime} b_{3} a_{1}+\delta_{2}^{\prime} b_{3} b_{6}^{3}}{a_{1} a_{6} a_{5}}+\frac{b_{1}^{\prime} b_{4}}{a_{2}}\right. \\
& \left.-\frac{a_{5}^{\prime} b_{4} b_{1}+b_{5}^{\prime} b_{1} a_{6}+\delta_{5}^{\prime} b_{1}^{3} b_{4}}{a_{2} a_{1} a_{6}}+\frac{b_{6}^{\prime} b_{3}}{a_{5}}\right] \text {, } \\
& t_{31}=\epsilon\left[-\frac{a_{2}^{\prime} b_{3} b_{6}-b_{2}^{\prime} b_{3} a_{6}+\delta_{2}^{\prime} b_{3} b_{6}^{3}}{a_{1} a_{6} a_{3}}+\frac{b_{1}^{\prime} b_{4}}{a_{4}}\right. \\
& \left.-\frac{a_{5}^{\prime} b_{4} b_{1}-b_{5}^{\prime} b_{1} a_{1}+\delta_{5}^{\prime} b_{1}^{3} b_{4}}{a_{4} a_{1} a_{6}}-\frac{b_{6}^{\prime} b_{3}}{a_{3}}\right] \text {, } \\
& f_{12}=\epsilon\left[-\frac{a_{1} a_{6}\left(b_{2}^{\prime} b_{3}^{2} a_{1}-b_{2}^{\prime} a_{6}-\delta_{2}^{\prime} a_{1} a_{6} b_{3}\right)-a_{3}^{2} b_{6}^{2}\left(a_{2}^{\prime} b_{3}+b_{2}^{\prime} a_{1}\right)}{a_{1} a_{6} a_{3} a_{5}}\right. \\
& \left.+\frac{b_{1}^{\prime}}{a_{2}}-\frac{a_{5}^{\prime} b_{1}+b_{5}^{\prime} a_{6} b_{1} b_{4}+\delta_{5}^{\prime} b_{1}}{a_{2} a_{1} a_{6}}+\frac{\left(a_{6}^{\prime}+\delta_{6}^{\prime}\right) b_{3}}{a_{3} a_{5}}\right] \text {, } \\
& f_{21}=\epsilon^{-1}\left[-\frac{a_{1} a_{6}\left(b_{2}^{\prime} b_{3}^{2} a_{6}+b_{2}^{\prime} a_{1}-\delta_{2}^{\prime} a_{1} a_{6} b_{3}\right)+a_{5}^{2} b_{6}^{2}\left(a_{2}^{\prime} b_{3}-b_{2}^{\prime} a_{6}\right)}{a_{1} a_{6} a_{3} a_{5}}\right. \\
& \left.+\frac{b_{1}^{\prime}}{a_{4}}+\frac{a_{5}^{\prime} b_{1}-b_{5}^{\prime} a_{1} b_{1} b_{4}+\delta_{5}^{\prime} b_{1}}{a_{4} a_{1} a_{6}}-\frac{\left(a_{6}^{\prime}+\delta_{6}^{\prime}\right) b_{3}}{a_{3} a_{5}}\right], \\
& f_{13}=\epsilon^{-1}\left[\frac{-b_{6}\left(b_{2}^{\prime} a_{6}+\delta_{2}^{\prime} a_{1} a_{6} b_{3}-a_{2}^{\prime} b_{3}\right)}{a_{1} a_{6} a_{3}}+\frac{b_{1}^{\prime} b_{4}}{a_{4}}\right. \\
& \left.-\frac{a_{1} b_{1}\left(b_{5}^{\prime}+\delta_{5}^{\prime} a_{6} b_{4}\right)-b_{1} b_{4} a_{5}^{\prime}}{a_{4} a_{1} a_{6}}+\frac{b_{6}^{\prime} b_{3}}{a_{3}}\right] \text {, } \\
& f_{31}=\epsilon\left[\frac{b_{6}\left(b_{2}^{\prime} a_{1}-\delta_{2}^{\prime} a_{1} a_{6} b_{3}+a_{2}^{\prime} b_{3}\right)}{a_{1} a_{6} a_{5}}-\frac{b_{1}^{\prime} b_{4}}{a_{2}}\right. \\
& \left.+\frac{a_{6} b_{1}\left(b_{5}^{\prime}-\delta_{5}^{\prime} a_{1} b_{4}\right)+b_{1} b_{4} a_{5}^{\prime}}{a_{2} a_{1} a_{6}}-\frac{b_{6}^{\prime} b_{3}}{a_{5}}\right] \text {, } \\
& f_{23}=\epsilon\left[-\frac{b_{6}\left(a_{2}^{\prime}+\delta_{2}^{\prime}+b_{2}^{\prime} a_{1} b_{3}\right)}{a_{1} a_{6} a_{5}}+\frac{\left(a_{1}^{\prime}-\delta_{1}^{\prime}\right) b_{4}}{a_{2} a_{4}}\right. \\
& \left.+\frac{a_{1} a_{6}\left(b_{5}^{\prime} a_{1}+\delta_{5}^{\prime} a_{1} a_{6} b_{4}-b_{5}^{\prime} a_{6} b_{4}^{2}\right)+a_{4}^{2} b_{1}^{2}\left(a_{5}^{\prime} b_{4}+b_{5}^{\prime} a_{6}\right)}{a_{1} a_{6} a_{2} a_{4}}+\frac{b_{6}^{\prime}}{a_{5}}\right] \text {, } \\
& f_{32}=\epsilon^{-1}\left[\frac{b_{6}\left(a_{2}^{\prime}+\delta_{2}^{\prime}-b_{2}^{\prime} a_{6} b_{3}\right)}{a_{1} a_{6} a_{3}}-\frac{\left(a_{1}^{\prime}-\delta_{1}^{\prime}\right) b_{4}}{a_{2} a_{4}}\right. \\
& \left.-\frac{a_{1} a_{6}\left(-b_{5}^{\prime} a_{6}+\delta_{5}^{\prime} a_{1} a_{6} b_{4}+b_{5}^{\prime} a_{1} b_{4}^{2}\right)+a_{2}^{2} b_{1}^{2}\left(a_{5}^{\prime} b_{4}-b_{5}^{\prime} a_{1}\right)}{a_{1} a_{6} a_{2} a_{4}}+\frac{b_{6}^{\prime}}{a_{3}}\right] \text {, } \\
& d_{11}=a_{3}^{\prime}+\frac{a_{2}^{\prime}}{a_{1} a_{6}}+\frac{a_{1}^{\prime}+\delta_{1}^{\prime} b_{4}^{2}}{a_{2} a_{4}}+\frac{b_{5}^{\prime} b_{4}\left(a_{1}-a_{6}\right)}{a_{1} a_{6} a_{2} a_{4}},
\end{aligned}
$$




$$
\begin{aligned}
d_{22} & =a_{2}^{\prime}+a_{1}^{\prime}+a_{5}^{\prime}+a_{6}^{\prime}+a_{4}^{\prime}+a_{3}^{\prime}, \\
d_{33} & =a_{4}^{\prime}+\frac{b_{2}^{\prime} b_{3}\left(a_{6}-a_{1}\right)}{a_{1} a_{6} a_{3} a_{5}}+\frac{a_{5}^{\prime}}{a_{1} a_{6}}+\frac{a_{6}^{\prime}+\delta_{6}^{\prime} b_{3}^{2}}{a_{3} a_{5}}, \\
d_{12} & =\frac{\delta_{5}^{\prime} a_{1} a_{6}-b_{2}^{\prime}\left(a_{1}-a_{1}\right) b_{3}}{a_{1} a_{6} a_{3} a_{5}}-\delta_{1}^{\prime}+\frac{a_{5}^{\prime}}{a_{1} a_{6}}+\frac{a_{6}^{\prime}}{a_{3} a_{5}}-\delta_{3}^{\prime}, \\
d_{23} & =\frac{a_{2}^{\prime}}{a_{1} a_{6}}+\frac{a_{1}^{\prime}}{a_{2} a_{4}}-\delta_{4}^{\prime}+\frac{b_{5}^{\prime} b_{4}\left(a_{6}-a_{1}\right)+\delta_{5}^{\prime} a_{1} a_{6}}{a_{1} a_{6} a_{2} a_{4}}-\delta_{6}^{\prime}, \\
d_{13} & =a_{2}^{\prime}+a_{1}^{\prime}+a_{5}^{\prime}+a_{6}^{\prime}, \\
d_{123} & =\frac{b_{2}^{\prime} a_{1} a_{6} b_{3}\left(a_{6}-a_{1}\right)-\delta_{2}^{\prime}-a_{2}^{\prime} b_{6}^{2}}{a_{1} a_{6} a_{3} a_{5}}-\frac{\delta_{1}^{\prime}}{a_{2} a_{4}} \\
& -\frac{a_{5}^{\prime} b_{1}^{2}+b_{5}^{\prime} a_{1} a_{6} b_{4}\left(a_{1}-a_{6}\right)-\delta_{5}^{\prime}}{a_{1} a_{6} a_{2} a_{4}}-\frac{\delta_{6}^{\prime}}{a_{3} a_{5}} .
\end{aligned}
$$

Acknowledgements: The authors A.S. and T.S. acknowledge the LAPTH for hospitality, where this work was carried out. T.S. acknowledge also SCOPE grant of SNF, Volkswagen foundation, INTAS grant 00561 and A.S INTAS grant 00-390 for partial financial support.

\section{References}

[1] J. Chalker, P. Coddington, J. Phys. C 21 (1988) 2665.

[2] A. Sedrakyan, Nucl.Phys.554 B [FS] (1999) 514.

[3] A. Sedrakyan, Integrable Chain Models with Staggered $R$-matrices, Contribution to the proceedings of Advanced NATO Workshop on Statistical Field Theories, Editors: A. Capelli, G. Mussardo, Como, June 18-23, 2001.

[4] D. Arnaudon, R. Poghossian, A. Sedrakyan, P. Sorba, Integrable Chain Model with Additional Staggered Model Parameter, Nucl. Phys. 588 B [FS] (2000) 638.

D. Arnaudon, R. Poghossian, A. Sedrakyan, T. Sedrakyan, and P. Sorba, "Construction of integrable models on ladders, and related quantum symmetries," in Non-perturbative Quantum Effects 2000, Paris, September 7-13, 2000 (B. Julia and D. Bernard, eds.), JHEP vol. PRHEP-tmr2000/053.

[5] J. Ambjorn, D. Arnaudon, A. Sedrakyan, T. Sedrakyan, and P. Sorba, Integrable ladder $t-J$ model with staggered shift of the spectral parameter, [hep-th/0006243], J. Phys. A: Math. Gen. 34 (2001) 5887-5900.

[6] T. Sedrakyan, Staggered anisotropy parameter modification of the anisotropic $t-J$ model, [nlin.SI/0103027], Nucl. Phys. 608 B [FS] (2001) 557-576.

[7] D. Arnaudon, A. Sedrakyan, T. Sedrakyan, and P. Sorba, Generalization of the $\mathcal{U}_{q}(g l(N))$ Algebra and Staggered Models, Lett. Math.Phys. 58 (2001) 209-222. 
[8] R.J. Baxter, Exactly Solved Models in Statistical Mechanics, Academic Press, London (1982).

[9] L. Faddeev, L. Takhtajian, Russian Math. Surveys 34:5 (1979) 11

V. Korepin, N. Bogoliubov, A. Izergin, Quantum Inverse Scattering Method and Correlation Functions, Cambridge Univ.Press (1993).

[10] M. F. Weiss, K. D. Schotte, Lattice Approach to the Spectrum of the Massive Thirring Model, Nucl. Phys. 225 B (1983) 247-260.

[11] C. Destri, H. J. De Vega, Light-cone Lattice Approach to Fermionic Theories in 2D. The Massive Thirring Model, Nucl. Phys. 290 B (1987) 363-391.

[12] V.Yu. Popkov, A.A. Zvyagin, Phys.Lett. A 175 (1993) 295,

A.A. Zvyagin, Phys.Rev. 51 B (1995) 12579.

[13] Y. Wang, Phys.Rev. B 60 (1999) 9236,

M.T. Batchelor, M. Maslen, J. Phys. A 32 (1999) L377,

M.T. Batchelor, J. de Gier, J. Links and M. Maslen, J. Phys. A 33 (2000) L97.

[14] A.K. Kolezhuk, H.-J. Mikeska, Int. J. Mod. Phys. B 12(1998) 2325,

A. Honecker, F. Mila, M. Troyer, cond-mat/9910438.

[15] N. Muramoto, M. Takahashi, J. Phys. Soc. Japan 68(1999) 2098.

[16] S. Albeverio, S.-M. Fei, Y. Wang, Europhys. Lett. 47(1999) 364.

[17] J. Links, A. Foerster, Solution of a two leg spin ladder system, cond-mat/9911096.

[18] L. D. Faddeev, N.Yu. Reshetikhin and L.A. Takhtajan, Quantization of Lie groups and Lie algebras, Leningrad Math. J. 1 (1990) 193.

[19] V.F.R. Jones, Baxterisation, Int. J. Mod. Phys. B4 (1990) 701, proceedings of "YangBaxter equations, conformal invariance and integrability in statistical mechanics and field theory", Canberra, 1989.

[20] J. Ambjorn, D. Karakhanyan, M. Mirumyan, A. Sedrakyan, Fermionization of the Spin-S Uimin-Lai-Sutherland Model: Generalization of Supersymmetric $t-J$ Model to Spin-S, Nucl. Phys. 599 B (2001), 547-560.

[21] F. Berezin, The Method of Second Quantization(Nauka, Moscow 1965),

L. Faddeev, Introduction to Functional Methods, in Les Houches(1975)

Session 28, ed. R. Balian, J. Zinn-Justin.

[22] N. Reshetikhin, Multiparameter quantum groups and twisted quasitriangular Hopf algebras, Lett. Math. Phys. 20 (1990) 331.

V.K. Dobrev, Duality for the matrix quantum group $G L_{p, q}(2, \mathbb{C})$, J. Math. Phys. 33 (1992) 3419. 